\title{
Coupled myovascular expansion directs growth and regeneration of the neonatal
} mouse heart

Paige DeBenedittis $\mathrm{PhD}^{1}$, Anish Karpurapu ${ }^{1}$, Albert Henry MD MSc ${ }^{2,3}$, Michael C Thomas $^{1}$, Timothy J McCord ${ }^{1}$, Kyla Brezitski ${ }^{1}$, Anil Prasad ${ }^{1}$, Yoshihiko Kobayashi PhD ${ }^{4}$, Svati H Shah MD MHS ${ }^{1}$, Christopher D Kontos MD ${ }^{1,5}$, Purushothama Rao Tata PhD ${ }^{4,6,7}$, R Thomas Lumbers MD PhD ${ }^{3,8,9}$, and Ravi Karra MD MHS $1,6,7,10$

${ }^{1}$ Division of Cardiology, Department of Medicine, Duke University Medical Center, Durham, NC, USA

${ }^{2}$ Institute of Cardiovascular Science, University College London, London, UK

${ }^{3}$ Institute of Health Informatics, University College London, London, UK

${ }^{4}$ Department of Cell Biology, Duke University, Durham, NC, USA

${ }^{5}$ Department of Pharmacology \& Cancer Biology, Duke University, Durham, NC, USA

${ }^{6}$ Regeneration Next, Duke University, Durham, NC, USA

${ }^{7}$ Center for Aging, Duke University Medical Center, Durham, NC, USA

${ }^{8}$ Health Data Research UK London, University College London, UK

${ }^{9}$ British Heart Foundation Research Accelerator, University College London, UK

${ }^{10}$ Department of Pathology, Duke University Medical Center, Durham, NC, USA

Address correspondence to: Ravi Karra MD MHS

Box 102152 DUMC

Durham, NC 27710

ravi.karra@duke.edu 


\section{ABSTRACT}

Innate heart regeneration in zebrafish and neonatal mammals requires multiple cell types, such as epicardial cells, nerves, and macrophages, to enable proliferation of spared cardiomyocytes (CMs). How these cells interact to create growth niches is unclear. Here we profile proliferation kinetics of cardiac endothelial cells (CECs) and CMs in the neonatal mouse heart and find that CM and CEC expansion is spatiotemporally coupled. We show that coupled myovascular expansion during cardiac growth or regeneration is dependent upon VEGF-VEGFR2 signaling, as genetic deletion of Vegfr2 from CECs or inhibition of VEGFA abrogates both CEC and CM proliferation. Repair of cryoinjury, a model of incomplete regeneration, displays poor spatial coupling of CEC and CM proliferation. Boosting CEC density in the border zone by injection of virus encoding Vegfa enhances $\mathrm{CM}$ proliferation and the efficacy of heart regeneration, suggesting that revascularization strategies to increase CEC numbers may be an important adjunct for approaches designed to promote CM proliferation after injury. Finally, we use a human Mendelian randomization study to demonstrate that circulating VEGFA levels are positively associated with higher myocardial mass among healthy individuals, suggesting similar effects on human cardiac growth. Our work demonstrates the importance of coupled CEC and CM expansion for cardiomyogenesis and reveals the presence of a myovascular niche that underlies cardiac growth and regeneration. 


\section{INTRODUCTION}

In many organs, heterologous cell types establish specialized microenvironments, or niches, that mediate tissue growth and regeneration. Alterations to niche constituents can affect the efficiency of tissue growth and outcomes after injury, making niches a possible target for therapeutic regeneration (Lane et al., 2014; Wagers, 2012). For organs like the intestines, bone marrow, skin, and skeletal muscle, niches are classically centered around a stem cell compartment (Fuchs and Blau, 2020). With a few notable exceptions, niches within organs that lack a resident stem cell are less defined. For instance, in the developing and regenerating neonatal mouse heart, hypoxic niches have been associated with regionalized growth, but the cellular makeup of these niches is unclear (Kimura et al., 2015).

Studies in zebrafish, salamanders, and neonatal mice have established a template for innate heart regeneration through proliferation of spared cardiomyocytes (CMs) (Jopling et al., 2010; Kikuchi et al., 2010; Oberpriller and Oberpriller, 1971; Poss et al., 2002). However, innate heart regeneration is clearly a multicellular process with required contributions from epicardial cells, inflammatory cells, and nerves (Aurora et al., 2014; Mahmoud et al., 2015; Wang et al., 2015). Recent work has highlighted a critical role for the vasculature in heart regeneration (Fernandez et al., 2018; Liu et al., 2020; Marin-Juez et al., 2016). In zebrafish and neonatal mice, cardiac endothelial cells (CECs) rapidly respond to injury, extending nascent vessels into the wound that ultimately guide $\mathrm{CM}$ growth (Das et al., 2019; Marin-Juez et al., 2019; Marin-Juez et al., 2016). Lineage tracing studies have demonstrated that these new vessels form by proliferation of CECs (Das et al., 2019; Marin-Juez et al., 2019; Zhao et al., 2014). Functional interference with 
angiogenic responses in the zebrafish or the neonatal mouse heart is associated with defects in CM proliferation (Das et al., 2019; Marin-Juez et al., 2016). Conversely, overexpression of the master angiogenic factor, vegfaa, is sufficient to induce ectopic cardiac growth in zebrafish, suggesting that a stimulated vasculature instructs cardiac growth (Karra et al., 2018). However, a similar role for stimulated CECs in the mammalian heart has yet to be shown.

Here, we spatiotemporally model CM and CEC proliferation in the neonatal mouse heart to investigate the mechanisms underlying cardiac growth and regeneration. We find that $\mathrm{CM}$ and $\mathrm{CEC}$ proliferation are tightly coupled during postnatal cardiac growth. With cryoinjury $(\mathrm{Cl})$, a model of incomplete regeneration, this coupling is spatially impaired. We demonstrate that coupled myovascular expansion is dependent on VEGFA signaling to endothelial VEGFR2 and restoration of coupling after Cl through exogenous Vegfa can enhance regeneration. Similarly, we find that genetically-determined circulating levels of VEGFA are associated with higher myocardial mass in humans. Together, these data demonstrate that coupled expansion of CECs and CMs within a myovascular niche regulates cardiac growth and regeneration.

\section{RESULTS}

\section{CEC and $\mathrm{CM}$ proliferation are spatiotemporally coupled during postnatal growth.}

Neonatal mice are able to regenerate their hearts after injury during the first few days of life (Porrello et al., 2011). The loss of regenerative capacity in the mouse heart is coincident with a developmental decline in the rate of CM proliferation. While the kinetics of $\mathrm{CM}$ proliferation in neonatal mice have been well-documented, dynamics of other 
cardiac cell types are not well characterized. To better define the relationship of CMs and CECs during the regenerative window of the neonatal mouse heart, we assayed CM and CEC proliferation kinetics at various time points following an EdU pulse (Figure 1a-b). Cardiac sections at different developmental time points were stained for EdU incorporation along with PCM1 and Erg to specifically mark CM and CEC nuclei, respectively (Alkass et al., 2015; Bergmann et al., 2009; Bergmann et al., 2011; Das et al., 2019). We developed customized image segmentation routines to objectively quantify large numbers of CECs and CMs from cardiac sections (3120 \pm 734 CECs per heart and $2328 \pm 751$ CMs per heart, mean \pm SD, see Quantification of CM and CEC Proliferation under Methods). Consistent with prior reports, we found the percentage of $\mathrm{dU}^{+} \mathrm{CMs}$ to sharply decline over the first 10 days of life, with a second peak occurring at P5 (Figure 1b,d). The increase in $\mathrm{EdU}^{+} \mathrm{CMs}$ at P5 likely coincides with a terminal round of DNA synthesis and binucleation of CMs (Alkass et al., 2015; Soonpaa et al., 1996). Proliferation of CECs also declines from P1 to P10 (Figure 1a,c) with a second peak at P7. While CM and CEC kinetics differ with regards to the timing of this second peak, the overall trends of their kinetics parallel each other. To determine the strength of this relationship, we compared rates of $\mathrm{EdU}^{+} \mathrm{CECs}$ and $\mathrm{CMs}$ for individual hearts and found them to be strongly correlated $(R=0.59, p=0.0007)$ (Figure 1e). Above a threshold of $\sim 8 \% \mathrm{EdU}^{+}$CECs, each $1 \%$ increase in $\mathrm{EdU}^{+} \mathrm{CMs}$ is associated with a $1.03 \pm 0.27 \%(\mathrm{p}=$ 0.0007) increase in EdU ${ }^{+}$CECs.

Based on the strong temporal association of CEC and CM proliferation, we next sought to spatially relate CEC and CM proliferation. Within thick sections of hearts from P4 mice pulsed with EdU, we observed numerous instances of EdU ${ }^{+} \mathrm{CMs}$ adjacent to 
$\mathrm{EdU}^{+}$CECs (Figure 1f). To quantify this observation, we assigned coordinates to each CEC and CM nucleus and computed pairwise distances for every CEC and CM. We used this distance information to deconvolve overlapping microenvironments by mapping the position of every CEC relative to an $\mathrm{EdU}^{+} \mathrm{CM}$ or an EdU- $\mathrm{CM}$ as a function of distance, resulting in "pseudodistance maps" (Figure 1g). When comparing the density of EdU+ CECs relative to an $\mathrm{EdU}^{+} \mathrm{CM}$ or EdU- $\mathrm{CM}$, we found the percentage of EdU ${ }^{+} \mathrm{CEC}$ s to be enriched around $\mathrm{EdU}^{+} \mathrm{CMs}$ compared to EdU- CMs until about $\sim 7 \mu \mathrm{m}$ (Figure $1 \mathrm{~g}-\mathrm{i}$ ), providing evidence for coupled myovascular expansion during physiologic growth.

\section{Coupling of myovascular growth after cryoinjury to the neonatal mouse heart.}

Unlike other models of injury to the neonatal mouse heart, cryoinjury $(\mathrm{Cl})$ results in incomplete or inefficient regeneration (Bakovic et al., 2020; Darehzereshki et al., 2015; Polizzotti et al., 2016; Polizzotti et al., 2015). Detailed analyses of CM proliferation kinetics after $\mathrm{Cl}$ have demonstrated that $\mathrm{CM}$ proliferation occurs in this model, but ostensibly at levels lower than that of uninjured neonatal heart (Darehzereshki et al., 2015; Polizzotti et al., 2015). Thus, we chose to evaluate myovascular expansion following $\mathrm{Cl}$ at $\mathrm{P} 1$ as a model of inefficient regeneration, in a regeneration-competent context. We first profiled CM and CEC kinetics, considering on average of $2407 \pm 891$ CECs and $2153 \pm 1495$ CMs within the border zone for each heart (mean $\pm \mathrm{SD}$ ). We found that $\mathrm{CM}$ and CEC kinetics generally follow the same trend as in the uninjured heart, with an approximately $50 \%$ decrease in proliferation indices for both cell types over the first 10 days of life (Figure 2 a-d). When we compared rates of CM and CEC proliferation in individual hearts, we once again noted that $\mathrm{CM}$ and CEC proliferation rates are correlated with $\sim 1 \%$ 
increase in the percentage of EdU ${ }^{+}$CECs for every percent increase of EdU ${ }^{+} \mathrm{CMs}(\mathrm{R}=$ $0.54, p=0.008)$ (Figure 2e). However, when we performed a spatial analysis of CECs and CMs within the border zone, we did not detect enrichment of EdU ${ }^{+}$CECs in the immediate vicinity of EdU ${ }^{+} \mathrm{CMs}$ (Figure $2 \mathrm{~g}, \mathrm{~h}$ ). In fact, EdU ${ }^{+}$CECs may even be depleted around $\mathrm{EdU}^{+} \mathrm{CMs}$ in the border zone after $\mathrm{Cl}$, suggesting that $\mathrm{CEC}$ and $\mathrm{CM}$ proliferation may not be efficiently coupled after $\mathrm{Cl}$.

\section{VEGFA-VEGFR2 signaling is associated with myovascular coupling.}

To better understand the molecular mediators of myovascular coupling during growth and injury, we performed single cell RNA-sequencing (scRNA-Seq) using border zones of P7 hearts that were cryoinjured at P1. We profiled 1721 cells and identified 9 clusters of cells carrying markers of CECs, CMs, fibroblasts, and inflammatory cells (Supplemental Table 1, Figure 3a). Clusters 1, 4, and 6 were notable for cells with CEC markers, such as Fabp4, Pecam1, Erg, and Vegfr2 (Supplemental Table 1). Compared to the other CEC clusters, cluster 4 had increased expression of numerous proliferative markers, including Ki67, Prc1, Ccna2, and Ccnb2 (Supplemental Table 2). A similar cluster of highly proliferative CECs has been also reported by other groups after coronary ligation in neonatal and adult mice (Wang et al., 2020; Wu et al., 2020). In the neonatal mouse heart, VEGFR2 marks cardiac microvascular cells and these cells have recently been implicated in revascularization following injury (Das et al., 2019). Evaluation of sections from neonatal hearts under physiologic growth conditions and after injury revealed multiple clusters of EdU ${ }^{+} \mathrm{CMs}$ in close proximity to EdU $\mathrm{VEGFR2}^{+} \mathrm{CECs}$ (Figure 3b, c), 
supporting a role for VEGFR2 ${ }^{+}$microvascular CECs as contributing to a myovascular niche of growth.

For many organs, parenchymal cells secrete angiogenic factors that enable matching of vascular supply to organ size (Rafii et al., 2016). Based on our prior work linking vegfaa overexpression to ectopic cardiomyogenesis in the zebrafish heart, we assayed Vegfa expression during growth and regeneration by single molecule fluorescent in situ hybridization (Karra et al., 2018). We found that under physiologic growth conditions, Vegfa is expressed in CMs but at low levels (Supplemental Figure 1a). After injury, Vegfa is sharply upregulated throughout the ventricle (Supplemental Figure 1b). However, based on our time course of $\mathrm{Cl}$ responses, CEC proliferation wanes in the border zone over time (Figure 2a). To determine whether Vegfa levels may be contributing to $\mathrm{CEC}$ dynamics after $\mathrm{Cl}$, we performed quantitative in situ hybridization of the border zone at P3 and P10 in hearts cryoinjured at P1 (Erben and Buonanno, 2019). We found Vegfa expression to decline by $\sim 50 \%$ from P3 to P10 in the border zone (Figure $3 \mathrm{~d}, \mathrm{e})$, paralleling kinetics of CEC proliferation. Together, these data support a dynamic role for myocardial VEGFA to endothelial VEGFR2 signaling as a regulator of the myovascular expansion during growth and regeneration.

\section{Endothelial Vegfr2 is required for $\mathrm{CM}$ proliferation during growth and regeneration.}

Based on our scRNA-Seq experiments indicating that many proliferating CECs are Vegfr2 ${ }^{+}$, we hypothesized that VEGFR2 signaling is a critical mediator of myovascular growth in the early neonatal period. To conditionally delete Vegfr2 from CECs, we crossed Vegfr2 flox/flox mice to Cdh5-CreER $R^{\top 2}$ mice to generate Cdh5-CreER ${ }^{\top 2} ;$ Vegfr2 flox/flox 
$\left(\right.$ Vegfr $\left.^{\Delta \mathrm{EC}}\right)$ and Vegfr $2^{f l o x}$ fllox $_{\left(V e g f r 2^{W T}\right.}$ ) mice (Hooper et al., 2009; Sorensen et al., 2009).

We verified loss of VEGFR2 from CECs by immunostaining for VEGFR2 in Vegfr2 ${ }^{\triangle E C}$ mice (Supplementary Figure 2). Evaluation of CECs in Vegfr2 ${ }^{\triangle E C}$ mice revealed fewer CECs than in Vegfr ${ }^{W T}$ animals and a nearly $80 \%$ reduction in CEC proliferation (Figure 4 a,b). To determine how CM growth is affected by the absence of Vegfr2 from CECs, we next assayed EdU incorporation by CMs. While CM numbers were largely preserved, CM cycling was also attenuated by $\sim 80 \%$ in Vegfr $2^{\triangle \mathrm{EC}}$ mice compared to Vegfr ${ }^{W T}$ mice (Figure $4 \mathrm{c}, \mathrm{d}$ ). We next evaluated the effect of Vegfr2 deletion from CECs on myovascular growth after cryoinjury. Similar to our results during early neonatal growth, relative numbers of CECs were decreased while relative CM numbers were preserved in Vegfr $^{\triangle \mathrm{EC}}$ hearts. We identified defects in both $\mathrm{CEC}$ and $\mathrm{CM}$ proliferation after injury in Vegfr $^{\triangle E C}$ mice (Figure 4 e-h). Specifically, we observed Vegfr2 ${ }^{\triangle E C}$ hearts to have an almost $80 \%$ decrease in cycling CECs after injury and a $40 \%$ decrease in cycling CMs. Consistent with our hypothesis, CM expansion during growth and regeneration is dependent on intact Vegfr2 in CECs.

\section{Inhibition of VEGFA limits CEC and CM proliferation.}

Our expression data suggest a role for myocardial Vegfa in myovascular coupling during growth and after injury (Figure S1, 3d). To functionally test this concept, we obtained a well-described synthetic antibody, B20-4.1.1 (anti-VEGFA), that binds murine VEGFA and prevents interaction with its receptors (Liang et al., 2006). Treatment of neonatal mice with anti-VEGFA decreased CEC proliferation by approximately $70 \%$ during early neonatal growth and by about $90 \%$ after injury indicating a dependency of CEC 
proliferation on VEGFA (Figure 5 a,b,e,f). Defects in CEC proliferation following antiVEGFA treatment were accompanied by decreases in the fraction of $\mathrm{EdU}^{+} \mathrm{CMs}$ during growth and after injury (Figure $5 \mathrm{c}, \mathrm{d}, \mathrm{g}, \mathrm{h}$ ), further supporting the need for myovascular coupling during growth and regeneration.

Prior work has described a critical role for tissue hypoxia as a regulator of CM proliferation in both zebrafish and mice (Jopling et al., 2012; Kimura et al., 2015; Puente et al., 2014). Remarkably, hypoxic pre-conditioning of adult mice results in cardiac growth and enhanced regenerative capacity (Nakada et al., 2017). Mechanistically, hypoxia decreases levels of reactive oxygen species, enabling CM cell cycle-reentry (Puente et al., 2014). As the decreased vascularity in Vegfr2 ${ }^{\triangle E C}$ mice and anti-VEGFA treated mice might be expected to result in tissue hypoxia, we examined hypoxia in CMs under these conditions. Pimonidazole is a 2-nitroimidazole used to identify tissue hypoxia based on its formation of stable adducts in the presence of low oxygen tension (Miller et al., 1989; Raleigh et al., 1998). These adducts can be detected by immunofluorescence with the intensity of staining directly proportional to the level of hypoxia. We administered pimonidazole prior to harvest of hearts from Vegfr ${ }^{\triangle E C}$ mice, Vegfr ${ }^{W T}$ mice, and mice injected with anti-VEGFA or vehicle. We noted an approximately $75 \%$ increase in the intensity of pimonidazole uptake by CMs of $V e g f r 2^{\triangle E C}$ mice compared to Vegfr2 ${ }^{W T}$ mice and an almost 2-fold increase in mice treated with anti-VEGFA compared to mice treated with vehicle (Supplemental Figure 3). Together, these results suggest that CECs are a required mediator for $\mathrm{CM}$ proliferation in response to tissue hypoxia.

\section{Exogenous VEGFA can enhance the efficiency of innate regenerative responses.}


Based on the impaired spatial myovascular coupling of CECs and CMs after $\mathrm{Cl}$ (Figure 2g-i) and the decrease of Vegfa in the border zone after Cl (Figure 3d), we hypothesized that increasing Vegfa levels within the border zone might enhance the efficiency of regenerative growth following $\mathrm{Cl}$ (Karra et al., 2018). To test our hypothesis, we generated adeno-associated virus to overexpress Vegfa (AAV-Vegfa) or GFP (AAVGFP). We then cryoinjured mice at $\mathrm{P} 1$ and injected AAV-Vegfa or AAV-GFP into the border zone immediately after injury (Supplemental Figure 2b). Gross examination of AAV-Vegfa hearts compared to AAV-GFP hearts at P21 identified increased vascularity at the site of injury (Figure 6a). In addition, we noted that AAV-Vegfa hearts had $~ 50 \%$ less scarring of the left ventricle and better cardiac function after $\mathrm{Cl}$ (Figure $6 \mathrm{~b}-\mathrm{d}$ ), all suggestive of enhanced regeneration. Importantly, while CEC density was increased in animals treated with AAV-Vegfa (Figure 6 e,f), this was accompanied by a more than 2fold increase of $\mathrm{Ki} 67^{+} \mathrm{CMs}$ in the border zone compared to hearts treated with AAV-GFP (Figure 6h). Together, these data indicate that exogeneous Vegfa in the border zone can enhance regenerative responses after neonatal $\mathrm{Cl}$.

\section{Genetically predicted circulating VEGFA levels are associated with human myocardial mass phenotypes}

Based on the stimulatory effects of Vegfa on neonatal heart growth, we next sought to evaluate whether VEGFA might have similar effects in humans. To test the hypothesis that VEGFA effects cardiac growth, we performed two-sample cis Mendelian randomization (MR) analysis with genetically predicted circulating VEGFA concentration as the exposure variable and traits of cardiac structure and function as the outcomes 
(Figure 7a) (Davey Smith and Hemani, 2014). Under this framework, genetic variants acting in cis that associate with protein abundance (protein quantitative trait loci, $p Q T L$ ) are used as instrumental variables to estimate the unconfounded causal effects of the protein on the outcomes of interest. Given that genotype is determined by random allocation, according to Mendel's second law, MR estimates based on genetic instruments tend not to be systematically associated with confounding factors and are not prone to reverse causation whereby the outcome influences the exposure (Schmidt et al., 2020).

Genetic variants within the VEGFA gene region were selected from genetic association summary statistics from a genome-wide association study (GWAS) of directly measured circulating VEGFA concentration in over 30,000 individuals (Supplemental Table 3), (Folkersen et al., 2020). Variant association statistics for six parameters of left ventricular (LV) structure and function were then extracted from a GWAS of cardiac magnetic resonance imaging measures in the UK Biobank (Aung et al., 2019). A summary of the GWAS is presented in Supplemental Table 4. We applied these instruments, using a two-sample MR model that accounts for partial correlation between instruments, to estimate the effect of genetically predicted circulating VEGFA on LV phenotypes (Schmidt et al., 2020). We did not detect a link between genetically predicted VEGFA levels and left ventricular volumes or ejection fraction but found that higher genetically predicted VEGFA levels resulted in increased myocardial mass (at type I error rate $=0.05 / 6$ ), (Figure $7 b)$. In total, we found an estimated $1.02(95 \% \mathrm{Cl}=0.33-1.70, p=0.004)$ gram increase in LVM per doubling of the circulating VEGFA concentration. These results are consistent with the hypothesis that VEGFA promotes cardiac growth in humans. 


\section{DISCUSSION}

We profiled CEC and CM dynamics to determine that CEC and CM proliferation are spatiotemporally coupled in the neonatal mouse heart. Uncoupling CECs from CMs, by deletion of Vegfr2 from CECs or with an anti-VEGFA antibody, dramatically decreases CM proliferation (Figures 4 and 5). Conversely, improving $\mathrm{CEC}$ and $\mathrm{CM}$ coupling after $\mathrm{Cl}$ by increasing local Vegfa increases regeneration after $\mathrm{Cl}$ (Figure 6). Analogously, myocardial mass of the human heart increases with higher VEGFA levels (Figure 7). Together, our work demonstrates that the efficiency of cardiac growth is dependent upon myovascular interactions, a finding with strong translational relevance. Approximately $50 \%$ of patients with systolic heart failure have flow-limiting coronary artery disease (Benjamin et al., 2018). Even in so-called "non-ischemic" cardiomyopathies, cardiac perfusion is impaired due to microvascular disease and capillary rarefaction (Abraham et al., 2000; Drakos et al., 2010; Mosseri et al., 1991; Parodi et al., 1993; Tsagalou et al., 2008). Thus, as methods to promote proliferation of adult mammalian CMs move towards therapeutics, mechanical or molecular revascularization approaches may be a required adjunct for these approaches to be maximally efficacious.

A key concept suggested by our work is that of growth niches made up of CECs and CMs throughout the heart (Figure 1,2). We find that CEC proliferation is statistically enriched within $7 \mu \mathrm{m}$ of proliferating CMs during physiologic growth. Because we used pairwise comparisons of CEC and CM distances to deconvolve overlapping niches, our spatial analysis likely underestimates the expanse of the niche surrounding proliferating CMs. However, our results are remarkably well-aligned with reported increases of CM 
proliferation within 3 cell lengths of sprouting angiogenesis in cultured fetal heart sections (Miao et al., 2020). Niches for CM expansion in the postnatal mammalian heart have been previously identified around hypoxia, with hypoxia even being able to induce CM proliferation in the adult mouse heart (Kimura et al., 2015; Nakada et al., 2017). Mechanistically, hypoxia exerts its effect on CM proliferation cell autonomously, by modulating reactive oxygen species and the DNA damage response (Puente et al., 2014). Our work suggests another element to the phenomenon of hypoxia-induced CM proliferation. Using two different approaches, genetic deletion of Vegfr2 from CECs and an anti-VEGFA antibody, we find that CM proliferation is attenuated without CECs despite an increase in myocardial hypoxia. Thus, hypoxia mediated CM expansion is likely to involve both CM-specific effects but to also depend on other cell types within these niches. Because therapeutic hypoxia for patients with heart failure may be challenging, a better understanding of the signaling milieu of the hypoxic niche could lead to potential therapeutics.

Because depletion of CECs, either through deletion of Vegfr2 from CECs or by administration of anti-VEGFA, leads to defects in CM proliferation, our work demonstrates that CECs promote CM expansion (Figure 5,6). Mechanistically, CEC expansion may simply lead to new conduits for growth factors or other cell types that promote cardiac growth. However, multiple lines of evidence also point to a direct role for CECs to influence CM proliferation through angiocrines. Indeed, recent work in zebrafish and neonatal mice support this concept. In zebrafish, inhibition of Notch in CECs results in reduced secretion of Wnt inhibitors that influence CM proliferation (Zhao et al., 2019). More directly, deletion of Igf2 from CECs in developing mice abrogates CM proliferation 
after neonatal cardiac injury (Shen et al., 2020). Interestingly, deletion of Igf2 from CECs does not affect physiologic CM proliferation, raising the intriguing possibility that different angiocrines modulate myocardial expansion during physiologic growth and after injury. Finally, different CEC subsets may contribute different sets of angiocrines, as recent work suggests that Reln from lymphatic endothelial cells has direct effects on CM proliferation (Liu et al., 2020). Future work to identify the complement of angiocrines that instruct CM proliferation in different developmental and injury contexts is needed.

Our work also suggests that restoring myovascular coupling can enhance regenerative capacity. Unlike physiologic growth, CEC proliferation is not enriched around proliferating $\mathrm{CMs}$ in the border zone after $\mathrm{Cl}$, a model of incomplete regeneration. Functionally increasing CEC density through overexpression of Vegfa enhances CM proliferation and reduces scarring after $\mathrm{Cl}$, both signs of enhanced regeneration (Figure 6). Taken together with our prior work showing that vegfaa overexpression can induce ectopic heart regeneration in zebrafish, exogenous Vegfa may be one approach to augment regeneration. Our Mendelian randomization study, in fact, predicts efficacy of VEGFA to increase myocardial mass (Figure 7). However, clinical trials of Vegfa to increase myocardial perfusion and reduce angina in patients with coronary heart disease have not been successful (Taimeh et al., 2013). One potential explanation for these trial results is that crude delivery methods including direct injection of plasmid and the use of early generation virus did not sufficiently raise local Vegfa levels (Oh and Ishikawa, 2019; Taimeh et al., 2013). Since that time, elegant methods for Vegfa delivery have evolved including the use of newer viral vectors, modified RNA, or engineered nanofibers (Lin et al., 2012; Zangi et al., 2013). Several clinical trials of Vegfa (NCT03409627, 
NCT03370887, and NCT03370887) are now underway to reassess molecular revascularization of the heart using these newer delivery methods, with possible regenerative implications.

In summary, here, we present work showing that coordinated growth of CECs and CMs guides postnatal cardiac growth and regeneration. We speculate that a better understanding of the signaling milieu within a myovascular niche can inform approaches for heart regeneration. 


\section{METHODS}

Mice and Neonatal Cryoinjuries

All protocols were approved by the Institutional Animal Care and Use Committee at Duke University. CD-1 mice (Charles River Labs, Morrisville, NC) were used for profiling myovascular kinetics during growth and regeneration. CD-1 mice were also used for antiVEGFA antibody and AAV experiments. Cdh5-CreER ${ }^{\mathrm{T} 2}$ and $V$ egfr2 ${ }^{\text {flox/flox }}$ strains have been previously described (Hooper et al., 2009; Sorensen et al., 2009). Cdh5-CreER ${ }^{\top 2}$; Vegfr2 $2^{\text {floxflox }}$ and Vegfr2 ${ }^{\text {flox/flox }}$ mice were treated with $100 \mu \mathrm{g}$ of tamoxifen intraperitoneally at $\mathrm{PO}$ and $\mathrm{P} 1$. Complete recombination was verified by immunostaining for VEGFR2 (Supplementary Figure 2). For proliferation studies, $0.5 \mathrm{mg}$ EdU was given intraperitoneally 4 hours prior to tissue harvest.

Cryoinjuries were performed on P1 pups as previously described (Bakovic et al., 2020; Polizzotti et al., 2015).

\section{Histology and Immunostaining}

At the time of tissue harvest, hearts were perfused with $\mathrm{KCl}$ and then $4 \%$ PFA. Hearts were then immersed in $30 \%$ sucrose overnight and embedded in Tissue Freezing Media for cryosectioning.

Cryosections were blocked with PBST (PBS with 0.1\% Tween-20) containing 10\% newborn calf serum and 1\% DMSO and incubated overnight with primary antibodies at $4^{\circ} \mathrm{C}$. Cryosections were then washed with PBST and incubated with secondary antibodies and DAPI (100 ng/ml). For EdU detection, cryosections were incubated in EdU staining solution (100 mM Tris-HCl, $1 \mathrm{mM} \mathrm{CuSO}_{4}, 10 \mu \mathrm{M}$ Azide, and $50 \mathrm{mM}$ ascorbic acid in PBS) 
for 10 minutes. Primary antibodies used for this study included: anti-PCM1 (Sigma, HPA023370, 1:100); anti-PCM1 (Santa Cruz, sc-398365, 1:100); anti-Erg (Abcam, ab92513, 1:25); anti-CD31 (BDBioscience, 553370, 1:100), anti-VEGFR2 (BDBioscience, 555307, 1:100), anti-Ki67 (ThermoFisher, 4-5698-82, 1:100), anti-Actinin (Cell Signaling, 6487P, 1:100). Secondary antibodies and azides were conjugated to Alexa-488, Alexa-594, or Alexa-633 (Invitrogen). To label CM nuclei, cryosections underwent heat induced epitope retrieval with citrate buffer prior to immunostaining. Stained slides were imaged on a Zeiss Axiolmager M1 epifluorescent microscope, a Zeiss CSU-X1 spinning disk confocal microscope, or a Zeiss LSM 510 confocal microscope. For physiologic growth experiments, 9 -16 non-overlapping images (40X) of the left ventricle were obtained for each section. For Cl experiments, the border zone was defined as the entire region within 1-2 40X fields of view along the entirety of the injury plane. For each heart, the 3 largest sections were imaged.

\section{Quantification of CM and CEC Proliferation}

We developed several customized image segmentation pipelines using CellProfiler and Ilastik for automated scoring of CEC and CM proliferation (Berg et al., 2019; Lamprecht et al., 2007). For CEC quantification, grayscale images for each channel were processed with rolling-ball background subtraction and used as input into a CellProfiler routine that 1) identified $\mathrm{Erg}^{+}$nuclei based on Erg staining and DAPI intensity; 2) identified EdU ${ }^{+}$nuclei based on EdU staining and DAPI staining; and 3) identified $\mathrm{EdU}^{+} \mathrm{Erg}^{+}$nuclei based on the presence of an EdU object within an Erg object. 
For CM proliferation, grayscale images from individual channels were obtained using a Zeiss CSU-X1 spinning disk confocal microscope. Images were pre-processed to generate 32-bit grayscale images and to create a set of images with merged PCM1 and DAPI channels. The DAPI image and the merged PCM1-DAPI images were then input into machine learning routines to generate probability maps for $\mathrm{DAPl}^{+}$nuclei and $\mathrm{PCM}^{+} / \mathrm{DAPI}^{+}$nuclei, using llastik. Machine learning algorithms were trained using images from our physiologic growth experiments. Probability maps and grayscale images were used as input for a CellProfiler pipeline that 1) identified nuclei based on a DAPI probability map; 2) filtered nuclei for $\mathrm{CMs}$ based on the mean $\mathrm{PCM}^{+} / \mathrm{DAPI}^{+}$pixel probability; 3) identified EdU ${ }^{+}$nuclei based on EdU staining and DAPI staining; and 4) identified EdU+ CM nuclei based on the presence of an EdU object within a CM nucleus. CellProfiler output data was tabulated using the dplyr package in R (Wickham et al., 2015).

\section{Proximity mapping}

For proximity mapping, z-stack images of P4 hearts were obtained using a Zeiss LSM 510 confocal microscope. CM and CEC nuclei were segmented using the Spots tool along with manual refinement, assigned coordinates, and categorized as EdU+ or EdU- using Imaris (Bitplane AG, Zurich, Switzerland). Coordinate files were then used to determine the pairwise distance of each CM and each CEC nucleus. Distances were used to compute the density of proliferating CECs as a function of distance from each $\mathrm{PCM} 1^{+}$ nucleus using the dplyr and plotly packages in R (Sievert et al., 2018; Wickham et al., 2015). 
In situ hybridization

RNAscope Probe - Mm-Vegfa-O2 (Lot 16197A) was hybridized against cryosections using the manufacturer's protocol (Advanced Cell Diagnostics, Hayward, CA). Images of sections were quantified using a CellProfiler routine adapted from Erben et al (Erben and Buonanno, 2019). Briefly, the DAPI channel was used to identify nuclei objects and the number of Vegfa spots adjacent to each nucleus was counted.

Single Cell RNA Sequencing and Analysis

Border zones of cryoinjured hearts were collected at P7 and then dissociated into single cells. Single cells were then captured into droplets using microfluidics followed by preparation of single cell cDNA libraries as previously described (Kobayashi et al., 2020). Samples were sequenced on a single lane of an Illumina HiSeq. Sequencing reads were mapped to Ensembl release NCBIM37.67. Aligned reads were binned and collapsed onto cellular barcodes using the Drop-seq pipeline v1.13.3 (http://mccarrolllab.com/dropseq), resulting in a raw digital expression matrix containing the count of unique UMIs for each gene in each cell (Macosko et al., 2015).

Expression analysis was performed by the Duke Bioinformatics Shared Resource, using the Seurat package (v2.2.0) in R/Bioconductor (Butler et al., 2018). Cells with gene counts over 4,000 or less than 200 were removed. Genes expressed in less than three cells were also removed. To avoid over-filtering CMs, we did not filter cells based on high expression of mitochondrial genes. Gene expression, for 1721 cells across 12754 genes, was normalized by scaling by the total number of transcripts, multiplying by 10,000 , and 
log transformation. Unwanted sources of variation were adjusted for by regression on the number of detected UMls using the ScaleData function. We used the JackStraw method to determine the number of statistically significant principal components (PCs). The FindClusters function was used to identify cellular clusters, using the 20 significant PCs and a 0.6 resolution. We used the t-SNE method to visualize cells across 20 PCs in two dimensions. To identify cell types, we used the FindAIIMarkers function. Parameters were set to test all genes, expressed in at least $10 \%$ of the cells in each cluster, for differential expression.

\section{Anti-VEGFA Antibody Treatment}

Anti-VEGFA antibody (B20-4.1.1) was a kind gift from Genenetch (San Francisco, CA). Neonatal mice were injected with $5 \mu \mathrm{g} / \mathrm{g}$ of anti-VEGFA or vehicle at P0 and P4. Adequate injection of anti-VEGFA was confirmed by significant weight loss compared to control animals at the time of tissue harvest.

\section{Determination of Hypoxia}

Mice were given $60 \mu \mathrm{g} / \mathrm{g}$ pimonidazole (Hypoxyprobe, HP3-100kit) intraperitoneally 1.5 hours prior to tissue harvest. Tissues sections were stained with an anti-pimonidazole antibody (Hypoxyprobe, HP3-100kit, 1:100) and an antibody against $\alpha$-actinin. For each heart, 9 images of the left ventricle for 3 separate cardiac sections were obtained using an Axiolmager M1 microscope. Images were quantified using a CellProfiler routine to 1) create an image mask based on the $\alpha$-actinin stain and 2) determine the mean intensity of anti-pimonidazole stain. 


\section{AAV Generation and Treatment}

AAV encoding Vegfa-164 under the control of a CMV promoter was generated by PCR amplifying the Vegf164 isoform of Vegfa from a mouse endothelial cDNA library and cloning this fragment into the pTR2-eGFP with replacement of EGFP (Hewitt et al., 2009). AAV, serotype 9, was generated through the Duke Viral Vector core. AAV virus encoding GFP under the control of a CMV promoter was obtained from the Duke Viral Vector Core. For intracardiac injections, $12 \mu \mathrm{l}$ of $\mathrm{AAV}$ at $1 \times 10^{9} \mathrm{vg} / \mu \mathrm{l}$ was injected circumferentially around the injured area immediately after $\mathrm{Cl}$.

\section{Coronary vessel labeling}

The coronary vasculature was visualized by injection of a low viscosity polyurethane resin (PU4ii, VasQTec) into the apex of the left ventricle (Lee et al., 2019). Whole mount images were obtained using a stereoscope.

\section{Scar Assessment}

P21 hearts were serially sectioned from base to apex and stained with acid fuchsin orange G, as previously described (Karra et al., 2015). Stained slides were scanned with a Leica Aperio Digital Pathology Slide Scanner. Scar area as a percentage of the left ventricle was scored by a blinded reader. The average scar percentage across the 5 largest sections was determined for each heart. 


\section{Echocardiography}

Fractional shortening was determined by echocardiography using the Duke Cardiovascular Physiology Core. Images were analyzed by three blinded readers and an averaged reading is reported.

Mendelian Randomization to Determine Effects of Circulating VEGFA Levels on Human

\section{Cardiac Structure}

Two-sample MR analysis was performed using summary statistics from GWAS of circulating protein level measured with Olink proximity-extension assay in 30,931 individuals of European ancestries and GWAS of cardiac MRI - derived left ventricular phenotypes in 16,923 UK Biobank participants (Supplementary Table 4) (Aung et al., 2019; Folkersen et al., 2020). To represent effect in the original unit of measurements, the standardized estimates from both GWAS were back-transformed by multiplying the MR effect estimate with the estimated standard deviation of the traits. MR instruments for VEGFA were selected from variants that 1) are available in both GWAS, 2) located within the 200 kilobases flanking region from the genomic coordinate of VEGFA gene; 3) have a MAF $>0.01 ; 4)$ have a $P$-value for association with circulating VEGFA level $<1 \times 10^{-4}$; and 5) are LD-clumped to an $r^{2}$ threshold of 0.4 using plink with --clump option (Purcell et al., 2007). Mendelian randomization analysis was performed using an inverse-variance weighted model accounting for correlation between instruments, implemented in the MendelianRandomization R package (Burgess et al., 2016; Yavorska and Burgess, 2017). Correlation between instruments were estimated from a random sample of 10,000 UK Biobank participants of European ancestries (Bycroft et al., 2018). 


\section{Data Analysis}

All means are presented as mean \pm SEM and proportions as proportion $\pm 95 \%$ confidence interval $(95 \% \mathrm{Cl})$. Statistical analysis between two groups was performed using a twotailed unpaired t-test test. Pairwise comparisons between multiple groups were performed using a two-tailed unpaired t-test test with correction for multiple testing using Holm's method. A p $<0.05$ was set as an a priori threshold for significance. Statistical analysis and plots were generated in $\mathrm{R}$ using the dplyr, ggpubr, and ggplot2 packages (Kassambara, 2017; Wickham, 2011; Wickham et al., 2015). For each experiment, individual data points are presented in the plot and the sample size is specified in the figure legend.

Author contributions: PD, AK, AH, KB, AP, MCT, YK, RTL, and RK conducted experiments and acquired data. PD, SHS, PRT, RTL, and RK designed the experiments, analyzed data, and wrote the manuscript. TJM and CDK contributed unpublished reagents.

Acknowledgments: We would like to thank Ken Poss, Nenad Bursac, Doug Marchuk, and Howard Rockman for helpful comments and discussion. We would like to thank Dr. Helene Fradin Kirshner from the Duke Bioinformatics Shared Resource for assistance with the analysis of scRNA-seq data. This work was funded by R03 HL144812 (RK), a Duke University Strong Start Physician Scientist Award (RK), a Mandel Foundation Seed 
Grant (RK), the Walker P. Inman Endowment (RK), a UK Research and Innovation Rutherford Fellowship MR/S003754/1 hosted by Health Data Research UK (RTL), the BigData@Heart Consortium funded by the Innovative Medicines Initiative-2 Joint Undertaking under grant agreement No. 116074 (RTL), and the National Institute for Health Research University College London Hospitals Biomedical Research Centre (RTL). 


\section{FIGURE LEGENDS}

Figure 1. Myovascular coupling during early neonatal growth. (a) Representative images of sections from mice at postnatal day 1 (P1), 7 (P7), and 10 (P10). Sections are immunostained for Erg to mark CECs and EdU to identify proliferating CECs (arrows). (b) Representative sections from P1, P7, and P10 hearts immunostained for PCM1 to mark CMs and EdU to mark proliferating cells. Arrows point to EdU ${ }^{+} \mathrm{PCM} 1^{+}$proliferating $\mathrm{CMs}$. (c, d) Quantitation of CEC and CM proliferation in the early neonatal period ( $n=6$ mice per time point). Each gray point is an individual heart. ${ }^{*} p<0.05,{ }^{* *} p<0.01,{ }^{* * *} p<0.001$, ns $=$ not significant. $p$-values are the result of a two-sided $t$-test corrected for multiple comparisons using Holm's method. (e) Correlation of CEC and CM proliferation. Black line is the best-fit regression line and gray area indicates the $95 \% \mathrm{Cl} . \mathrm{p}$ value indicates significance of Pearson correlation. Each point represents an individual heart and is color coded by age. (f) Representative section from a P4 heart immunostained for PCM1, Erg, EdU, and DAPI. Boxed regions correspond to the adjacent magnified panels. Hollowed, yellow arrows are $\mathrm{PCM} 1^{+} \mathrm{EdU}{ }^{+} \mathrm{CMs}$ and solid yellow arrows are $\mathrm{Erg}^{+} \mathrm{EdU}{ }^{+} \mathrm{CECs}$. (g) Pseudodistance maps for detecting enrichment of $\mathrm{EdU}^{+} \mathrm{CECs}$ around $\mathrm{EdU}^{+} \mathrm{CMs}$ Distance information for 12,374 CEC-CM pairs from 6 mice was used to map EdU ${ }^{+}$CECs (red dots) and EdU- CECs (blue dots) relative to an averaged EdU ${ }^{-}$and EdU ${ }^{+} \mathrm{CM}$ (center of the plot). Each line represents $2 \mu \mathrm{m}$ from the center of an averaged CM nucleus. (h) Proportion of CEC's that are $E \mathrm{dU}^{+}$as a function of distance $(\mu \mathrm{m})$ from $\mathrm{PCM} 1^{+} \mathrm{EdU} \mathrm{U}^{+}$nuclei (red) and $\mathrm{PCM} 1^{+} \mathrm{EdU}-$ nuclei (blue). $274 \mathrm{EdU}^{+} \mathrm{CMs}$ and $3658 \mathrm{EdU}-\mathrm{CMs}^{-}$from 6 mice were considered. (i) Difference in the proportion of EdU ${ }^{+}$CECs within a $7 \mu \mathrm{m}$ radius of 
bioRxiv preprint doi: https://doi.org/10.1101/2021.01.20.425322; this version posted January 21, 2021. The copyright holder for this preprint (which was not certified by peer review) is the author/funder. All rights reserved. No reuse allowed without permission.

$\mathrm{PCM}^{+}{ }^{+} \mathrm{EUU}^{+}$and $\mathrm{PCM} 1^{+}$EdU- nuclei, $\mathrm{p}=0.04$, two-sided Z-test. Error bars show the $95 \%$ Cl. Scale bars are $50 \mu \mathrm{m}$. 
Figure 2. Myovascular coupling after cryoinjury. (a, b) Representative images of sections from hearts collected at $\mathrm{P} 1, \mathrm{P} 7$, and $\mathrm{P} 10$ from mice that underwent cardiac cryoinjury $(\mathrm{Cl})$ at $\mathrm{P} 1$. Arrows point to $\mathrm{EdU}^{+} \mathrm{Erg}^{+}$proliferating $\mathrm{CECs}$ in $(\mathrm{a})$ and $\mathrm{EdU} \mathrm{PCM}^{+}$ proliferating CMs in (b). (c, d) Quantitation of CEC and CM proliferation in the early neonatal period after cryoinjury ( $n=5-6$ mice per time point). Each gray point is an individual heart. ${ }^{* *} p<0.01,{ }^{* * *} p<0.001$. $p$-values are the result of a two-sided t-test corrected for multiple comparisons using Holm's method. (e) Correlation of CEC and CM proliferation. Black line is the best-fit regression line and gray area indicates the $95 \% \mathrm{Cl}$. $p$ value indicates significance of Pearson correlation. Each point is an individual heart, color coded by age. (f) Representative section from the border zone of a cryoinjured P4 heart immunostained for PCM1, Erg, EdU, and DAPI. Boxed regions correspond to the adjacent magnified panels. Hollowed, yellow arrows are $\mathrm{PCM} 1^{+} \mathrm{EdU} \mathrm{U}^{+} \mathrm{CMs}$ and solid yellow arrows are $\mathrm{Erg}^{+} \mathrm{EdU}^{+}$CECs. (g) Pseudodistance maps for detecting enrichment of EdU $^{+}$CECs around EdU ${ }^{+}$CMs. Distance information for 7,093 CEC-CM pairs from 6 mice post $\mathrm{Cl}$ was used to map EdU ${ }^{+}$CECs (red dots) and EdU- CECs (blue dots) relative to an averaged $\mathrm{EdU}^{-}$and $\mathrm{EdU}^{+} \mathrm{CM}$ (center of the plot). Each line represents $2 \mu \mathrm{m}$ from the center of an averaged CM nucleus. (h) Proportion of CEC's that are EdU ${ }^{+}$as a function of distance in $\mu \mathrm{m}$ from $\mathrm{PCM} 1^{+} \mathrm{EdU} U^{+}$nuclei (red) and PCM1 ${ }^{+} \mathrm{EdU}-$ nuclei (blue). Solid line indicates the point estimate. $186 \mathrm{EdU}^{+} \mathrm{CMs}$ and $2446 \mathrm{EdU}-\mathrm{CMs}$ were considered. (i) Difference in the proportion of EdU ${ }^{+}$CECs within a $7 \mu \mathrm{m}$ radius of $P C M 1^{+} E d U^{+}$and $\mathrm{PCM}^{+}{ }^{+}$EdU` nuclei, $\mathrm{p}=$ not significant (ns), two-sided Z-test. Error bars show the $95 \% \mathrm{Cl}$. Scale bars are $50 \mu \mathrm{m}$. 
Figure 3. VEGFA-VEGFR2 signaling is associated with myovascular coupling. (a) tSNE plots of scRNA-seq data from $\mathrm{P} 7$ hearts that were underwent $\mathrm{Cl}$ at $\mathrm{P} 1$. In the left most panel, cells are colored by clusters identified by PCA analysis. Arrows point to a cluster of CECs that are enriched for expression of Mki67 and Vegfr2 (purple). (b, c) Clusters of EdU ${ }^{+}$VEGFR2 ${ }^{+}$CECs adjacent to $\mathrm{EdU}^{+} \mathrm{Tnnt}^{+} \mathrm{CMs}$ at $\mathrm{P} 7$ during growth (b) and after $\mathrm{Cl}$ at $\mathrm{P} 1$ (c). Insets are magnifications of the regions in the solid white boxes. (d) Single molecule in situ hybridization for Vegfa in P3 and P10 hearts that underwent $\mathrm{Cl}$ at P1. Dashed line indicates the approximate injury plane, with the border zone above the line. Red marks Vegfa signal. (e) Quantification of in situ hybridization for Vegfa expression in the border zone of P3 $(n=5)$ and P10 $(n=5)$ hearts after $\mathrm{Cl}$ at $\mathrm{P} 1,{ }^{* *} \mathrm{p}=$ 0.008, two-sided t-test. Scale bars are $50 \mu \mathrm{m}$. 
Figure 4: Requirement of Vegfr2 in CECs for myovascular growth in the neonatal mouse heart. (a) Representative images of P4 hearts from Vegfr ${ }^{\mathrm{WT}}$ and Vegfr ${ }^{\mathrm{AEC}}$ mice, immunostained to detect cycling CECs. Arrows point to EdU ${ }^{+} \mathrm{Erg}^{+}$proliferating CECs. (b) Quantitation of CEC proliferation in $\operatorname{Vegfr}^{\mathrm{WT}}(n=5)$ and Vegfr ${ }^{\triangle \mathrm{EC}}$ mice $(n=6) .{ }^{* * * *} p=$ $3.7 \times 10^{-5}$, two-sided t-test. (c) Representative images of P4 hearts from Vegfr ${ }^{\mathrm{WT}}$ and Vegfr $^{\triangle \mathrm{EC}}$ mice, immunostained to detect cycling CMs. Arrows point to EdU ${ }^{+} \mathrm{PCM} 1^{+}$ proliferating CMs. (d) Quantitation of CM proliferation in Vegfr2 ${ }^{\mathrm{WT}}(\mathrm{n}=5)$ and $\operatorname{Vegfr}^{\mathrm{\Delta EC}}$ mice $(n=6) .{ }^{* *} p=0.0004$, two-sided t-test. (e) Images of $\mathrm{P} 4$ hearts from Vegfr2 ${ }^{\mathrm{WT}}$ and Vegfr2 ${ }^{\Delta E C}$ mice after $\mathrm{Cl}$ at $\mathrm{P} 1$. Sections are immunostained to detect cycling CECs. Arrows point to $\mathrm{EdU}^{+} \mathrm{Erg}^{+}$proliferating CECs. (f) Quantitation of CEC proliferation in $\operatorname{Vegfr}^{\mathrm{WT}}(\mathrm{n}=7)$ and Vegfr ${ }^{\mathrm{LEC}}$ mice $(n=8) .{ }^{* * *} \mathrm{p}=2.0 \times 10^{-5}$, two-sided t-test. $(\mathrm{g})$ Representative images of P4 hearts injured at P1 from Vegfr2 ${ }^{\mathrm{WT}}$ and Vegfr2 ${ }^{\mathrm{AEC}}$ mice. Sections are immunostained to detect cycling CMs. Arrows point to EdU ${ }^{+} P C M 1^{+}$ proliferating CMs. (h) Quantitation of CM proliferation in Vegfr2 ${ }^{\mathrm{WT}}(\mathrm{n}=7)$ and Vegfr2 ${ }^{\mathrm{AEC}}$ mice $(n=8) .{ }^{* *} p=0.004$, two-sided t-test. Scale bars are $50 \mu \mathrm{m}$. 
Figure 5: Requirement of VEGFA for myovascular growth in the neonatal mouse heart. (a) Representative images of P4 hearts from mice injected with vehicle or antiVEGFA. Sections are immunostained to detect cycling CECs. Arrows point to EdU+Erg ${ }^{+}$ proliferating CECs. (b) Quantitation of CEC proliferation at P4 after treatment with vehicle $(n=10)$ or anti-VEGFA $(n=5) .{ }^{* * * *} p=1.8 \times 10^{-6}$, two-sided t-test. (c) Representative images of P4 hearts after treatment with vehicle or anti-VEGFA. Sections are immunostained to detect cycling CMs. Arrows point to $\mathrm{EdU}{ }^{+} \mathrm{PCM} 1^{+}$proliferating $\mathrm{CMs}$. (d) Quantitation at P4 after treatment with vehicle $(n=10)$ or anti-VEGFA $(n=5) .{ }^{* *} p=0.003$, two-sided t-test. (e) Images of P4 hearts from mice that underwent $\mathrm{Cl}$ at $\mathrm{P} 1$ and that were injected with vehicle or anti-VEGFA. Arrows point to $\mathrm{EdU}^{+} \mathrm{Erg}^{+}$proliferating CECs. (f) Quantitation of CEC proliferation in Vegfr2WT $(n=4)$ and $\operatorname{Vegfr}{ }^{\Delta E C}$ mice $(n=3){ }^{* * * *} p=$ 6.9 $\times 10^{-5}$, two-sided t-test. (g) Immunostaining for PCM1 and EdU in P4 hearts from mice that underwent $\mathrm{Cl}$ at $\mathrm{P} 1$ and that were treated with vehicle or anti-VEGFA. Arrows point to $\mathrm{EdU}+\mathrm{PCM} 1^{+}$proliferating CMs. (h) Quantitation of CM proliferation after treatment with vehicle $(n=4)$ or anti-VEGFA $(n=3) .{ }^{* *} p=0.008$, two-sided t-test. Scale bars are $50 \mu \mathrm{m}$. 
Figure 6: Effect of Vegfa overexpression on regeneration after $\mathrm{Cl}$. (a) Whole mount images of representative P21 hearts, injected with either AAV-GFP or AAV-Vegfa at the time of $\mathrm{Cl}$ at $\mathrm{P} 1$. Blue resin opacifies the coronary vasculature. Circled area indicates the approximated area of injury. Scale bar is $1 \mathrm{~mm}$. (b) AFOG staining of P21 hearts to visualize scar (blue). Arrows indicate vessels and dashed line indicates the approximate area of injury. Scale bar is $500 \mu \mathrm{m}$. (c) Quantitation of scar as a percentage of the left ventricle at P21 in animals injected with AAV-GFP $(n=8)$ or AAV-Vegfa $(n=8)$ at the time of $\mathrm{Cl}$ at $\mathrm{P} 1 .{ }^{*} \mathrm{p}=0.038$. (d) Fractional shortening at $\mathrm{P} 21$ in animals injected with AAVGFP $(n=6)$ or AAV-Vegfa $(n=8)$ at the time of $\mathrm{Cl}$ at P1. ${ }^{* *} \mathrm{p}=0.008$, two-sided t-test. (e) Images of the border zone from $\mathrm{P} 10$ mice that underwent $\mathrm{Cl}$ at $\mathrm{P} 1$. Sections are immunostained to mark proliferating $\mathrm{Erg}^{+} \mathrm{Ki} 67^{+} \mathrm{CECs}$ (arrows). (f) Quantitation of CEC density at P14 after injection with AAV-GFP $(n=4)$ or AAV-Vegfa $(n=4) .{ }^{*} p=0.035$, twosided t-test. (g) Images of the border zone from P14 mice that underwent $\mathrm{Cl}$ at P1. Sections are immunostained to mark proliferating $\mathrm{PCM} 1^{+} \mathrm{Ki} 67^{+} \mathrm{CMs}$ (arrows). (h) Quantitation of CM proliferation at P14 after injection with AAV-GFP $(\mathrm{n}=4)$ or AAV-Vegfa $(n=4) .{ }^{*} p=0.017$, two-sided t-test. 
Figure 7: Effect of circulating VEGFA levels on human cardiac structure. (a) Schematic of Mendelian randomization (MR) analysis. (b) cis-Mendelian randomization estimate with inverse-variance weighted model of circulating VEGFA level on left ventricular end-diastolic volume (LVEDV), left ventricular end systolic volume (LVESV), left ventricular ejection fraction (LVEF), left ventricular mass to volume ratio (LVMR), and LV mass (LVM). Each data point represents effect estimate in standard deviation (SD) change of the trait per doubling the circulating VEGFA concentration. Error bars indicate $95 \%$ confidence intervals $(\mathrm{Cl})$ of the estimates. 
Figure S1. Vegfa expression in the neonatal mouse heart. (a-b) Tilescan images of an uninjured P3 heart (a) and a P3 heart that underwent $\mathrm{Cl}$ at P1 (b). Sections are immunostained for Tnnt (green) and hybridized with an in situ probe for Vegfa (red). Lines correspond to the approximate injury plane. The asterisks correspond to the magnified images. Scale bar is $500 \mu \mathrm{m}$.

Figure S2. Approaches to manipulate VEGFA/VEGFR2 signaling. (a) Images of Vegfr2 $^{\mathrm{WT}}$ and Vegfr ${ }^{\triangle \mathrm{EC}}$ mice at P3 after treatment with tamoxifen at P0. Sections are immunostained for PECAM and VEGFR2. The dashed box corresponds to magnified images, shown as individual channels. Scale bar is $100 \mu \mathrm{m}$. (b) Single molecule in situ hybridization for Vegfa in the border zone of P10 hearts that were injected with either AAV-GFP or AAV-Vegfa after $\mathrm{Cl}$ at P1. Dashed line indicates the approximate plane of injury, with the region above the line corresponding to the border zone. Red dots indicate Vegfa expression. Scale bar is $50 \mu \mathrm{m}$.

Figure S3: Myocardial hypoxia with loss of Vegfr2 in CECs or after administration of anti-VEGFA. (a) Images of sections from P4 Vegfr2 ${ }^{\mathrm{WT}}$ and Vegfr2 ${ }^{\Delta \mathrm{EC}}$ hearts immunostained for actinin (green) and pimonidazole (red) incorporation. (b) Quantitation of pimonidazole intensity per $\mu \mathrm{m}$ of tissue stained with actinin in $\operatorname{Vegfr}^{\mathrm{WT}}(\mathrm{n}=3)$ and $\operatorname{Vegfr}^{\triangle \mathrm{EC}}(\mathrm{n}=3)$ hearts. ${ }^{*} \mathrm{p}=0.011$, two-sided t-test. (c) Images of sections from P4 mice treated with vehicle or anti-VEGFA. Sections are immunostained for actinin (green) and pimonidazole (red) incorporation. (d) Quantitation of pimonidazole intensity per $\mu \mathrm{m}$ of 
bioRxiv preprint doi: https://doi.org/10.1101/2021.01.20.425322; this version posted January 21, 2021. The copyright holder for this preprint (which was not certified by peer review) is the author/funder. All rights reserved. No reuse allowed without permission.

tissue stained with actinin in mice treated with vehicle $(n=4)$ or anti-VEGFA $(n=4) .{ }^{* *} p=$ 0.005, two-sided t-test. Scale bars are $50 \mu \mathrm{m}$. 


\section{REFERENCES}

Abraham, D., Hofbauer, R., Schäfer, R., Blumer, R., Paulus, P., Miksovsky, A., Traxler, H., Kocher, A., and Aharinejad, S. (2000). Selective downregulation of VEGF-A165, VEGF-R1, and decreased capillary density in patients with dilative but not ischemic cardiomyopathy. Circulation research 87, 644-647.

Alkass, K., Panula, J., Westman, M., Wu, T.-D., Guerquin-Kern, J.-L., and Bergmann, O. (2015). No evidence for cardiomyocyte number expansion in preadolescent mice. Cell 163, 1026-1036.

Aung, N., Vargas, J.D., Yang, C., Cabrera, C.P., Warren, H.R., Fung, K., Tzanis, E., Barnes, M.R., Rotter, J.I., Taylor, K.D., et al. (2019). Genome-Wide Analysis of Left Ventricular Image-Derived Phenotypes Identifies Fourteen Loci Associated With Cardiac Morphogenesis and Heart Failure Development. Circulation 140, 1318-1330. Aurora, A.B., Porrello, E.R., Tan, W., Mahmoud, A.I., Hill, J.A., Bassel-Duby, R., Sadek, H.A., and Olson, E.N. (2014). Macrophages are required for neonatal heart regeneration. J Clin Invest 124, 1382-1392.

Bakovic, M., Thakkar, D., DeBenedittis, P., Chong, D.C., Thomas, M.C., Iversen, E.S., and Karra, R. (2020). Clonal Analysis of the Neonatal Mouse Heart using Nearest Neighbor Modeling. Journal of Visualized Experiments: Jove.

Benjamin, E.J., Virani, S.S., Callaway, C.W., Chamberlain, A.M., Chang, A.R., Cheng, S., Chiuve, S.E., Cushman, M., Delling, F.N., and Deo, R. (2018). Heart disease and stroke statistics-2018 update: a report from the American Heart Association.

Circulation.

Berg, S., Kutra, D., Kroeger, T., Straehle, C.N., Kausler, B.X., Haubold, C., Schiegg, M., Ales, J., Beier, T., and Rudy, M. (2019). llastik: interactive machine learning for (bio) image analysis. Nature Methods, 1-7.

Bergmann, O., Bhardwaj, R.D., Bernard, S., Zdunek, S., Barnabe-Heider, F., Walsh, S., Zupicich, J., Alkass, K., Buchholz, B.A., Druid, H., et al. (2009). Evidence for cardiomyocyte renewal in humans. Science 324, 98-102.

Bergmann, O., Zdunek, S., Alkass, K., Druid, H., Bernard, S., and Frisen, J. (2011). Identification of cardiomyocyte nuclei and assessment of ploidy for the analysis of cell turnover. Exp Cell Res 317, 188-194.

Burgess, S., Dudbridge, F., and Thompson, S.G. (2016). Combining information on multiple instrumental variables in Mendelian randomization: comparison of allele score and summarized data methods. Stat Med 35, 1880-1906.

Butler, A., Hoffman, P., Smibert, P., Papalexi, E., and Satija, R. (2018). Integrating single-cell transcriptomic data across different conditions, technologies, and species. Nat Biotechnol 36, 411-420.

Bycroft, C., Freeman, C., Petkova, D., Band, G., Elliott, L.T., Sharp, K., Motyer, A., Vukcevic, D., Delaneau, O., O'Connell, J., et al. (2018). The UK Biobank resource with deep phenotyping and genomic data. Nature 562, 203-209.

Darehzereshki, A., Rubin, N., Gamba, L., Kim, J., Fraser, J., Huang, Y., Billings, J., Mohammadzadeh, R., Wood, J., Warburton, D., et al. (2015). Differential regenerative capacity of neonatal mouse hearts after cryoinjury. Dev Biol 399, 91-99.

Das, S., Goldstone, A.B., Wang, H., Farry, J., D'Amato, G., Paulsen, M.J., Eskandari, A., Hironaka, C.E., Phansalkar, R., Sharma, B., et al. (2019). A Unique Collateral Artery 
Development Program Promotes Neonatal Heart Regeneration. Cell 176, 1128-1142 e1118.

Davey Smith, G., and Hemani, G. (2014). Mendelian randomization: genetic anchors for causal inference in epidemiological studies. Hum Mol Genet 23, R89-98.

Drakos, S.G., Kfoury, A.G., Hammond, E.H., Reid, B.B., Revelo, M.P., Rasmusson, B.Y., Whitehead, K.J., Salama, M.E., Selzman, C.H., and Stehlik, J. (2010). Impact of mechanical unloading on microvasculature and associated central remodeling features of the failing human heart. Journal of the American College of Cardiology 56, 382-391. Erben, L., and Buonanno, A. (2019). Detection and quantification of multiple RNA sequences using emerging ultrasensitive fluorescent in situ hybridization techniques. Current protocols in neuroscience 87, e63.

Fernandez, C.E., Bakovic, M., and Karra, R. (2018). Endothelial Contributions to Zebrafish Heart Regeneration. J Cardiovasc Dev Dis 5.

Folkersen, L., Gustafsson, S., Wang, Q., Hansen, D.H., Hedman, A.K., Schork, A., Page, K., Zhernakova, D.V., Wu, Y., Peters, J., et al. (2020). Genomic and drug target evaluation of 90 cardiovascular proteins in 30,931 individuals. Nat Metab 2, 1135-1148. Fuchs, E., and Blau, H.M. (2020). Tissue Stem Cells: Architects of Their Niches. Cell Stem Cell 27, 532-556.

Hewitt, F.C., Li, C., Gray, S.J., Cockrell, S., Washburn, M., and Samulski, R.J. (2009). Reducing the risk of adeno-associated virus (AAV) vector mobilization with AAV type 5 vectors. J Virol 83, 3919-3929.

Hooper, A.T., Butler, J.M., Nolan, D.J., Kranz, A., lida, K., Kobayashi, M., Kopp, H.G., Shido, K., Petit, I., Yanger, K., et al. (2009). Engraftment and reconstitution of hematopoiesis is dependent on VEGFR2-mediated regeneration of sinusoidal endothelial cells. Cell Stem Cell 4, 263-274.

Jopling, C., Sleep, E., Raya, M., Marti, M., Raya, A., and Izpisua Belmonte, J.C. (2010). Zebrafish heart regeneration occurs by cardiomyocyte dedifferentiation and proliferation. Nature 464, 606-609.

Jopling, C., Suñé, G., Faucherre, A., Fabregat, C., and Izpisua Belmonte, J.C. (2012). Hypoxia induces myocardial regeneration in zebrafish. Circulation 126, 3017-3027. Karra, R., Foglia, M.J., Choi, W.Y., Belliveau, C., DeBenedittis, P., and Poss, K.D. (2018). Vegfaa instructs cardiac muscle hyperplasia in adult zebrafish. Proc Natl Acad Sci U S A 115, 8805-8810.

Karra, R., Knecht, A.K., Kikuchi, K., and Poss, K.D. (2015). Myocardial NF-kappaB activation is essential for zebrafish heart regeneration. Proc Natl Acad Sci U S A 112, $13255-13260$.

Kassambara, A. (2017). ggpubr:"ggplot2" based publication ready plots. R package version 0.1. 6 .

Kikuchi, K., Holdway, J.E., Werdich, A.A., Anderson, R.M., Fang, Y., Egnaczyk, G.F., Evans, T., Macrae, C.A., Stainier, D.Y., and Poss, K.D. (2010). Primary contribution to zebrafish heart regeneration by gata4(+) cardiomyocytes. Nature 464, 601-605.

Kimura, W., Xiao, F., Canseco, D.C., Muralidhar, S., Thet, S., Zhang, H.M., Abderrahman, Y., Chen, R., Garcia, J.A., Shelton, J.M., et al. (2015). Hypoxia fate mapping identifies cycling cardiomyocytes in the adult heart. Nature 523, 226-230. 
Kobayashi, Y., Tata, A., Konkimalla, A., Katsura, H., Lee, R.F., Ou, J., Banovich, N.E., Kropski, J.A., and Tata, P.R. (2020). Persistence of a regeneration-associated, transitional alveolar epithelial cell state in pulmonary fibrosis. Nat Cell Biol 22, 934-946. Lamprecht, M.R., Sabatini, D.M., and Carpenter, A.E. (2007). CellProfiler: free, versatile software for automated biological image analysis. Biotechniques 42, 71-75. Lane, S.W., Williams, D.A., and Watt, F.M. (2014). Modulating the stem cell niche for tissue regeneration. Nat Biotechnol 32, 795-803.

Lee, H.K., Widmayer, S.J., Huang, M.-N., Aylor, D.L., and Marchuk, D.A. (2019). Novel Neuroprotective Loci Modulating Ischemic Stroke Volume in Wild-Derived Inbred Mouse Strains. Genetics 213, 1079-1092.

Liang, W.-C., Wu, X., Peale, F.V., Lee, C.V., Meng, Y.G., Gutierrez, J., Fu, L., Malik, A.K., Gerber, H.-P., and Ferrara, N. (2006). Cross-species vascular endothelial growth factor (VEGF)-blocking antibodies completely inhibit the growth of human tumor xenografts and measure the contribution of stromal VEGF. Journal of Biological Chemistry 281, 951-961.

Lin, Y.D., Luo, C.Y., Hu, Y.N., Yeh, M.L., Hsueh, Y.C., Chang, M.Y., Tsai, D.C., Wang, J.N., Tang, M.J., Wei, E.I., et al. (2012). Instructive nanofiber scaffolds with VEGF create a microenvironment for arteriogenesis and cardiac repair. Sci Transl Med 4, 146 ra109.

Liu, X., De la Cruz, E., Gu, X., Balint, L., Oxendine-Burns, M., Terrones, T., Ma, W., Kuo, H.H., Lantz, C., Bansal, T., et al. (2020). Lymphoangiocrine signals promote cardiac growth and repair. Nature.

Macosko, E.Z., Basu, A., Satija, R., Nemesh, J., Shekhar, K., Goldman, M., Tirosh, I., Bialas, A.R., Kamitaki, N., Martersteck, E.M., et al. (2015). Highly Parallel Genome-wide Expression Profiling of Individual Cells Using Nanoliter Droplets. Cell 161, 1202-1214. Mahmoud, A.I., O'Meara, C.C., Gemberling, M., Zhao, L., Bryant, D.M., Zheng, R., Gannon, J.B., Cai, L., Choi, W.Y., Egnaczyk, G.F., et al. (2015). Nerves Regulate Cardiomyocyte Proliferation and Heart Regeneration. Dev Cell 34, 387-399.

Marin-Juez, R., El-Sammak, H., Helker, C.S.M., Kamezaki, A., Mullapuli, S.T., Bibli, S.I., Foglia, M.J., Fleming, I., Poss, K.D., and Stainier, D.Y.R. (2019). Coronary

Revascularization During Heart Regeneration Is Regulated by Epicardial and

Endocardial Cues and Forms a Scaffold for Cardiomyocyte Repopulation. Dev Cell 51, 503-515 e504.

Marin-Juez, R., Marass, M., Gauvrit, S., Rossi, A., Lai, S.L., Materna, S.C., Black, B.L., and Stainier, D.Y. (2016). Fast revascularization of the injured area is essential to support zebrafish heart regeneration. Proc Natl Acad Sci U S A 113, 11237-11242. Miao, Y., Tian, L., Martin, M., Paige, S.L., Galdos, F.X., Li, J., Klein, A., Zhang, H., Ma, N., and Wei, Y. (2020). Intrinsic Endocardial Defects Contribute to Hypoplastic Left Heart Syndrome. Cell Stem Cell 27, 574-589. e578.

Miller, G., Best, M., Franko, A., Koch, C., and Raleigh, J. (1989). Quantitation of hypoxia in multicellular spheroids by video image analysis. International Journal of Radiation Oncology* Biology* Physics 16, 949-952.

Mosseri, M., Schaper, J., Admon, D., Hasin, Y., Gotsman, M., Sapoznikov, D., Pickering, J., and Yarom, R. (1991). Coronary capillaries in patients with congestive cardiomyopathy or angina pectoris with patent main coronary arteries. Ultrastructural morphometry of endomyocardial biopsy samples. Circulation 84, 203-210. 
Nakada, Y., Canseco, D.C., Thet, S., Abdisalaam, S., Asaithamby, A., Santos, C.X., Shah, A.M., Zhang, H., Faber, J.E., Kinter, M.T., et al. (2017). Hypoxia induces heart regeneration in adult mice. Nature 541, 222-227.

Oberpriller, J., and Oberpriller, J.C. (1971). Mitosis in adult newt ventricle. J Cell Biol 49, 560-563.

Oh, J.G., and Ishikawa, K. (2019). Recent highlights and advances in cardiac gene therapy. Discov Med 28, 229-235.

Parodi, O., De Maria, R., Oltrona, L., Testa, R., Sambuceti, G., Roghi, A., Merli, M., Belingheri, L., Accinni, R., and Spinelli, F. (1993). Myocardial blood flow distribution in patients with ischemic heart disease or dilated cardiomyopathy undergoing heart transplantation. Circulation 88, 509-522.

Polizzotti, B.D., Ganapathy, B., Haubner, B.J., Penninger, J.M., and Kuhn, B. (2016). A cryoinjury model in neonatal mice for cardiac translational and regeneration research. Nat Protoc 11, 542-552.

Polizzotti, B.D., Ganapathy, B., Walsh, S., Choudhury, S., Ammanamanchi, N., Bennett, D.G., dos Remedios, C.G., Haubner, B.J., Penninger, J.M., and Kuhn, B. (2015).

Neuregulin stimulation of cardiomyocyte regeneration in mice and human myocardium reveals a therapeutic window. Sci Transl Med 7, 281 ra245.

Porrello, E.R., Mahmoud, A.I., Simpson, E., Hill, J.A., Richardson, J.A., Olson, E.N., and Sadek, H.A. (2011). Transient regenerative potential of the neonatal mouse heart.

Science 331, 1078-1080.

Poss, K.D., Wilson, L.G., and Keating, M.T. (2002). Heart regeneration in zebrafish. Science 298, 2188-2190.

Puente, B.N., Kimura, W., Muralidhar, S.A., Moon, J., Amatruda, J.F., Phelps, K.L., Grinsfelder, D., Rothermel, B.A., Chen, R., Garcia, J.A., et al. (2014). The oxygen-rich postnatal environment induces cardiomyocyte cell-cycle arrest through DNA damage response. Cell 157, 565-579.

Purcell, S., Neale, B., Todd-Brown, K., Thomas, L., Ferreira, M.A., Bender, D., Maller, J., Sklar, P., de Bakker, P.I., Daly, M.J., et al. (2007). PLINK: a tool set for wholegenome association and population-based linkage analyses. Am J Hum Genet 81, 559575.

Rafii, S., Butler, J.M., and Ding, B.S. (2016). Angiocrine functions of organ-specific endothelial cells. Nature 529, 316-325.

Raleigh, J.A., Calkins-Adams, D.P., Rinker, L.H., Ballenger, C.A., Weissler, M.C., Fowler, W.C., Jr., Novotny, D.B., and Varia, M.A. (1998). Hypoxia and vascular endothelial growth factor expression in human squamous cell carcinomas using pimonidazole as a hypoxia marker. Cancer Res 58, 3765-3768.

Schmidt, A.F., Finan, C., Gordillo-Maranon, M., Asselbergs, F.W., Freitag, D.F., Patel, R.S., Tyl, B., Chopade, S., Faraway, R., Zwierzyna, M., et al. (2020). Genetic drug target validation using Mendelian randomisation. Nat Commun 11, 3255.

Shen, H., Gan, P., Wang, K., Darehzereshki, A., Wang, K., Kumar, S.R., Lien, C.-L., Patterson, M., Tao, G., and Sucov, H.M. (2020). Mononuclear diploid cardiomyocytes support neonatal mouse heart regeneration in response to paracrine IGF2 signaling. Elife 9, e53071. 
Sievert, C., Parmer, C., Hocking, T., Chamberlain, S., Ram, K., Corvellec, M., and Despouy, P. (2018). Plotly: create interactive web graphics via 'plotly. js'. R package. version 4.7. 1; 2017. URL https://CRAN R-project org/package= plotly.

Soonpaa, M.H., Kim, K.K., Pajak, L., Franklin, M., and Field, L.J. (1996). Cardiomyocyte DNA synthesis and binucleation during murine development. American Journal of Physiology-Heart and Circulatory Physiology 271, H2183-H2189.

Sorensen, I., Adams, R.H., and Gossler, A. (2009). DLL1-mediated Notch activation regulates endothelial identity in mouse fetal arteries. Blood 113, 5680-5688.

Taimeh, Z., Loughran, J., Birks, E.J., and Bolli, R. (2013). Vascular endothelial growth factor in heart failure. Nat Rev Cardiol 10, 519-530.

Tsagalou, E.P., Anastasiou-Nana, M., Agapitos, E., Gika, A., Drakos, S.G., Terrovitis, J.V., Ntalianis, A., and Nanas, J.N. (2008). Depressed coronary flow reserve is associated with decreased myocardial capillary density in patients with heart failure due to idiopathic dilated cardiomyopathy. Journal of the American College of Cardiology 52, 1391-1398.

Wagers, A.J. (2012). The stem cell niche in regenerative medicine. Cell Stem Cell 10, 362-369.

Wang, J., Cao, J., Dickson, A.L., and Poss, K.D. (2015). Epicardial regeneration is guided by cardiac outflow tract and Hedgehog signalling. Nature 522, 226-230.

Wang, Z., Cui, M., Shah, A.M., Tan, W., Liu, N., Bassel-Duby, R., and Olson, E.N. (2020). Cell-Type-Specific Gene Regulatory Networks Underlying Murine Neonatal Heart Regeneration at Single-Cell Resolution. Cell Rep 33, 108472.

Wickham, H. (2011). ggplot2. Wiley Interdisciplinary Reviews: Computational Statistics 3, 180-185.

Wickham, H., Francois, R., Henry, L., and Müller, K. (2015). dplyr: A Grammar of Data Manipulation: https://CRAN. R-project org/package= dplyr.

Wu, X., Reboll, M.R., Korf-Klingebiel, M., and Wollert, K.C. (2020). Angiogenesis After Acute Myocardial Infarction. Cardiovasc Res.

Yavorska, O.O., and Burgess, S. (2017). MendelianRandomization: an R package for performing Mendelian randomization analyses using summarized data. Int $\mathrm{J}$ Epidemiol 46, 1734-1739.

Zangi, L., Lui, K.O., von Gise, A., Ma, Q., Ebina, W., Ptaszek, L.M., Spater, D., Xu, H., Tabebordbar, M., Gorbatov, R., et al. (2013). Modified mRNA directs the fate of heart progenitor cells and induces vascular regeneration after myocardial infarction. Nat Biotechnol 31, 898-907.

Zhao, L., Ben-Yair, R., Burns, C.E., and Burns, C.G. (2019). Endocardial Notch Signaling Promotes Cardiomyocyte Proliferation in the Regenerating Zebrafish Heart through Wnt Pathway Antagonism. Cell Rep 26, 546-554 e545.

Zhao, L., Borikova, A.L., Ben-Yair, R., Guner-Ataman, B., MacRae, C.A., Lee, R.T., Burns, C.G., and Burns, C.E. (2014). Notch signaling regulates cardiomyocyte proliferation during zebrafish heart regeneration. Proc Natl Acad Sci U S A 111, 14031408. 
bioRxiv preprint doi: https://doi.org/10.1101/2021.01.20.425322; this version posted January 21, 2021. The copyright holder for this preprint (which was not certified by peer review) is the author/funder. All rights reserved. No reuse allowed without permission.
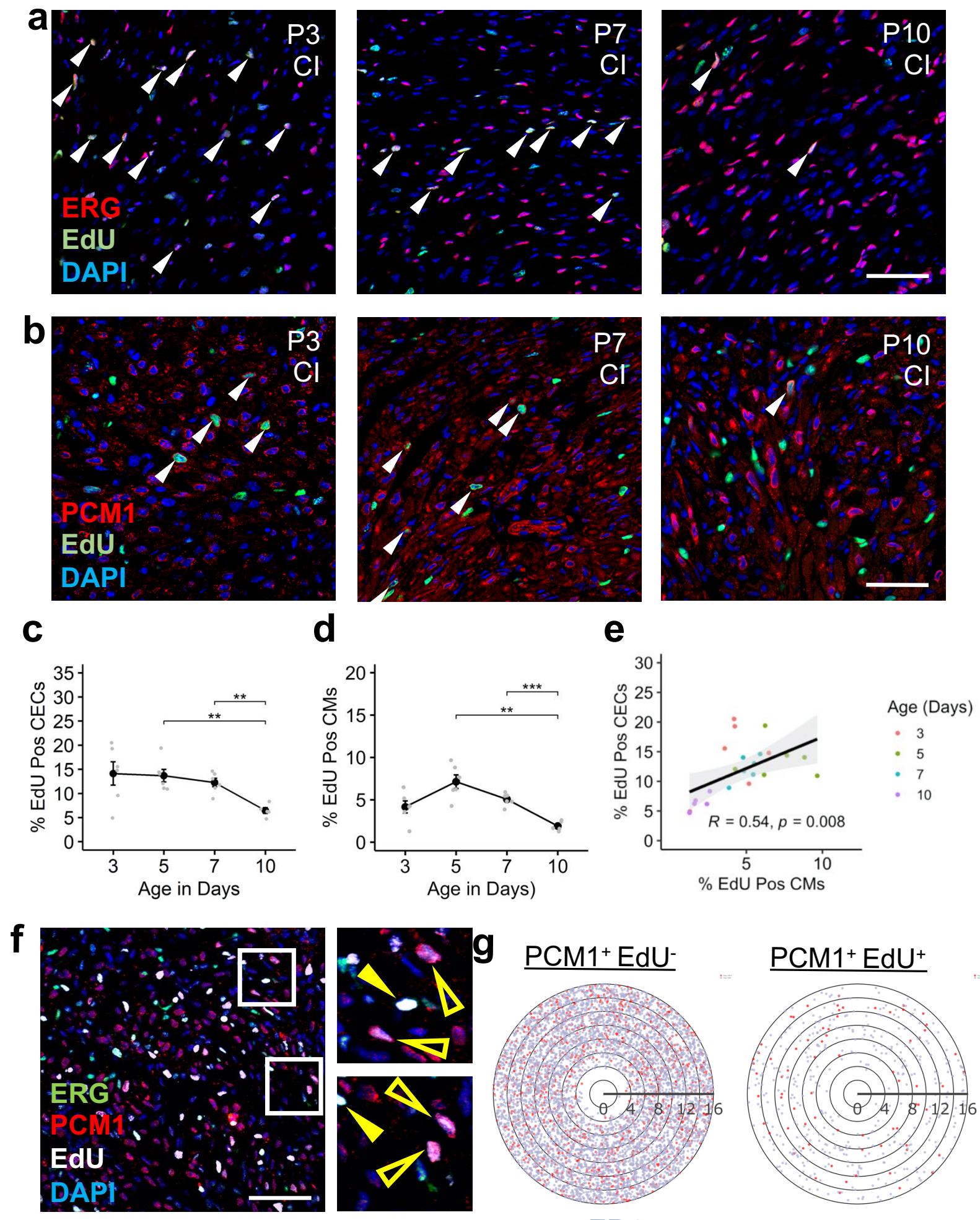

$\underline{\mathrm{PCM}^{+}{ }^{+} \mathrm{EdU}}+$

$E R G^{+} E d U-E R G+E d U^{+}$
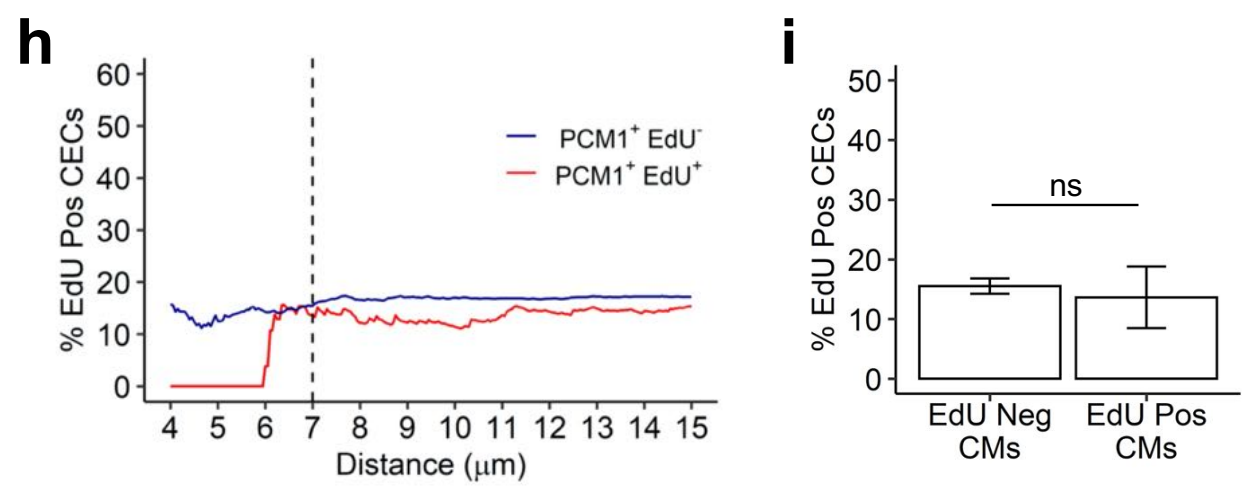

\section{Figure 2}




\section{Figure 3}

a

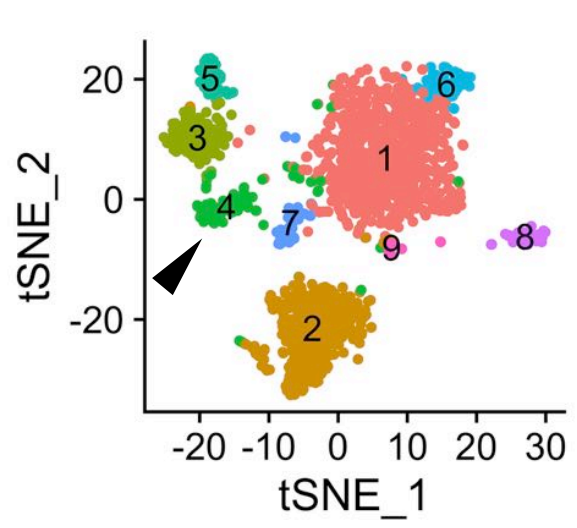

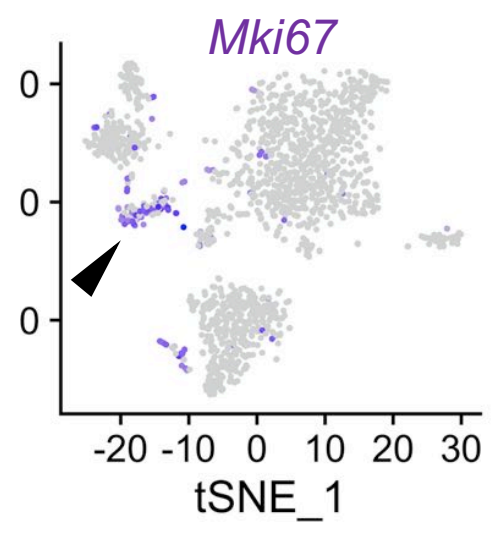

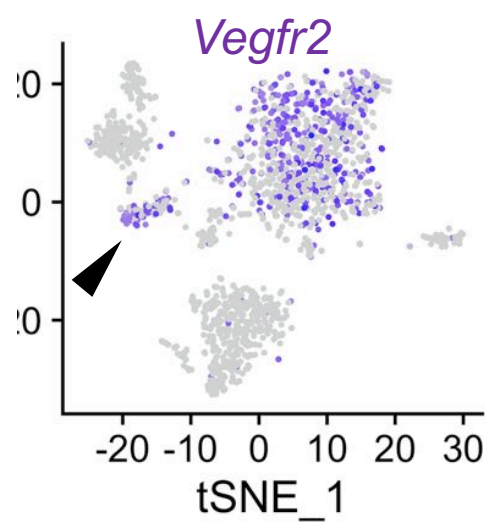

b

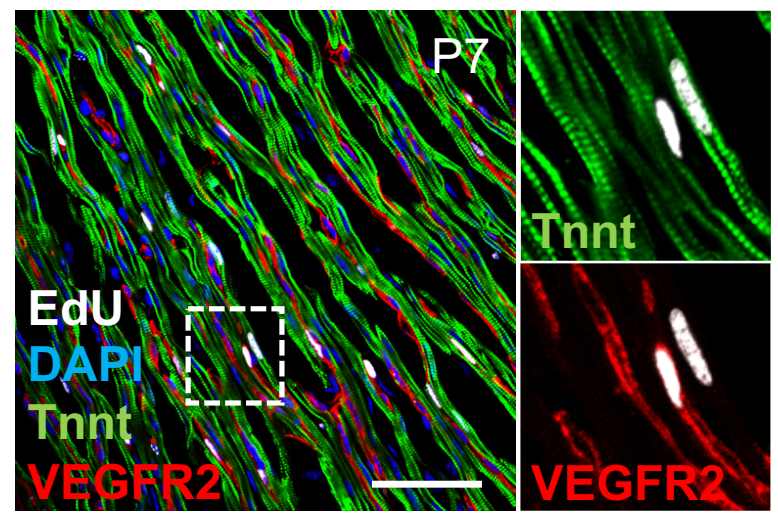

C

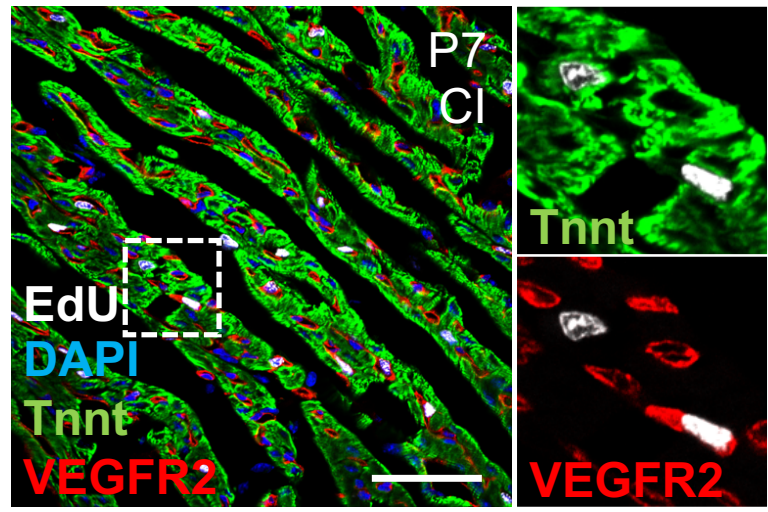

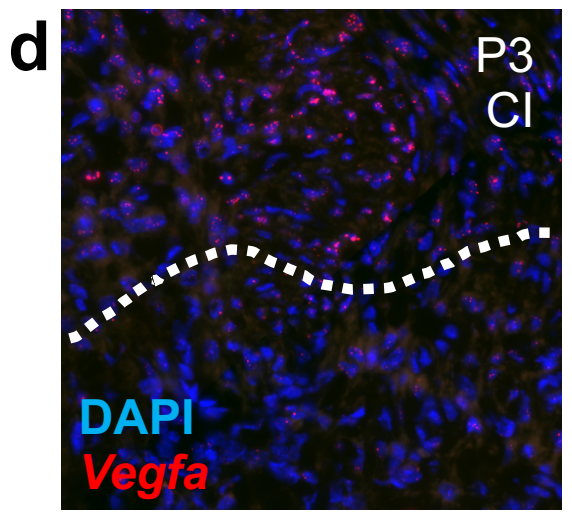
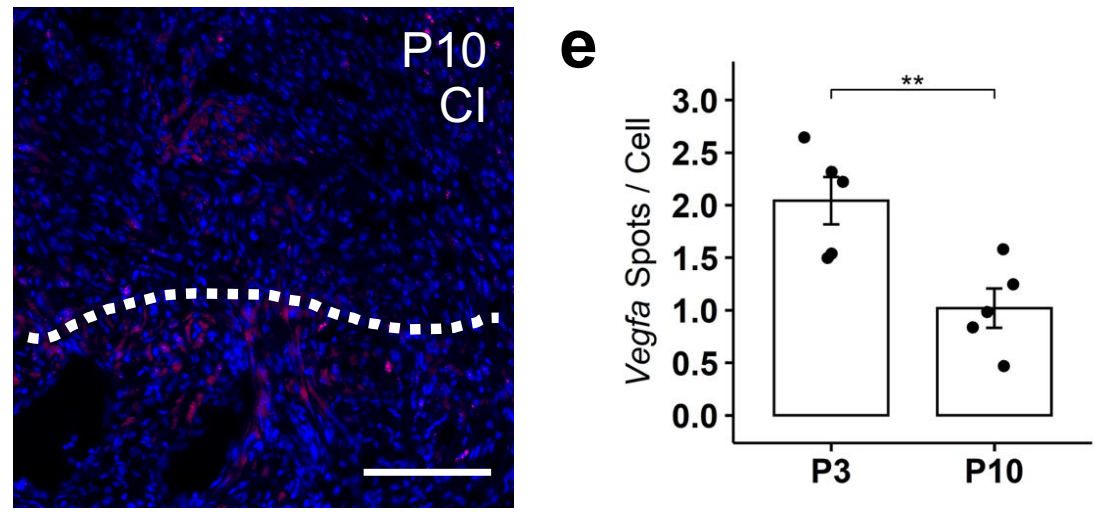
Physiologic Growth
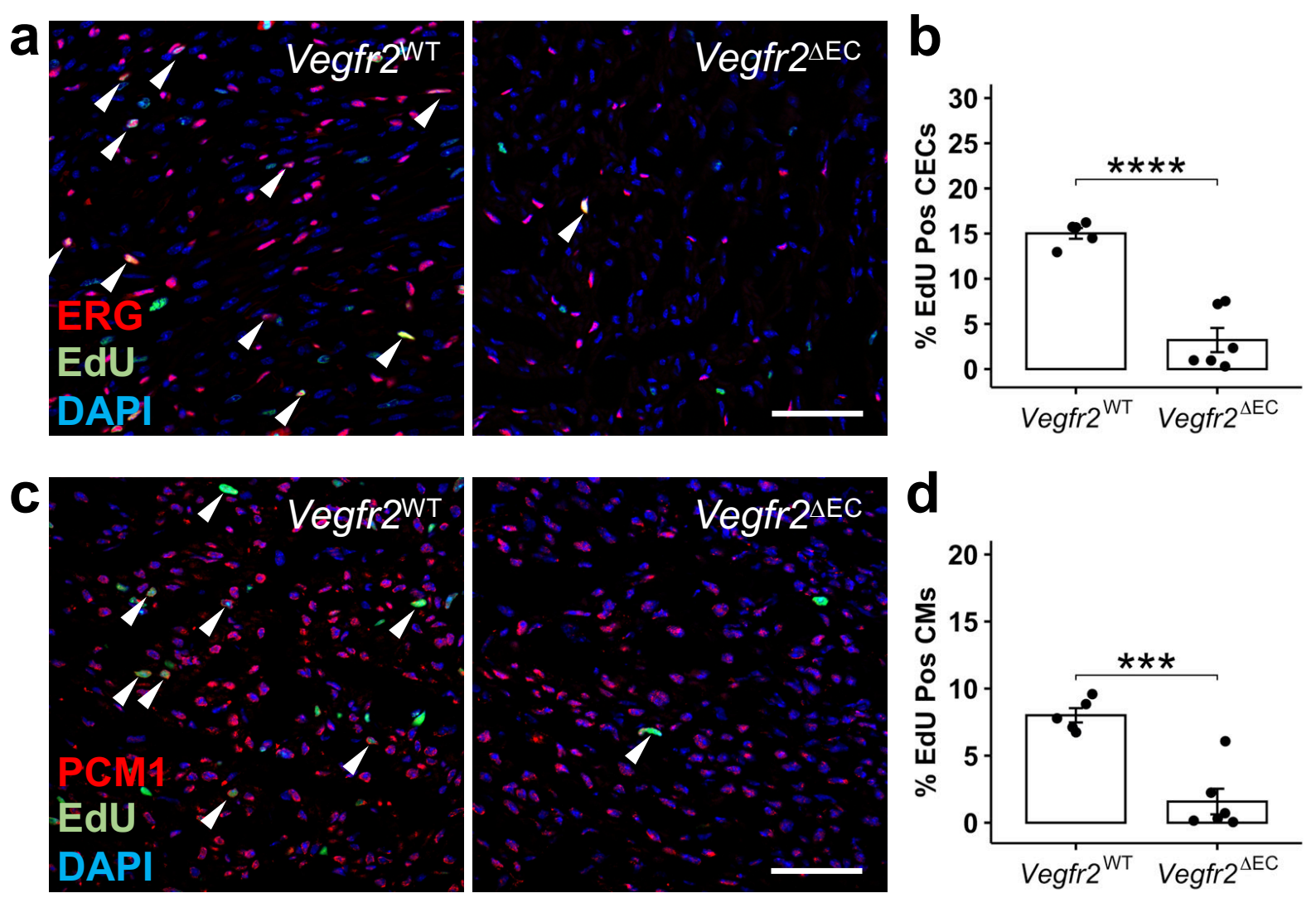

After Injury

e

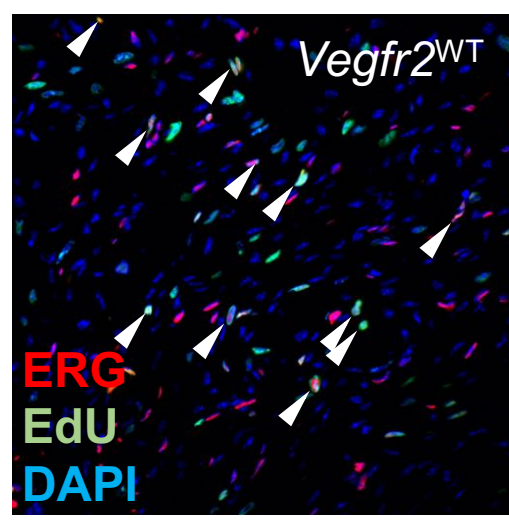

g

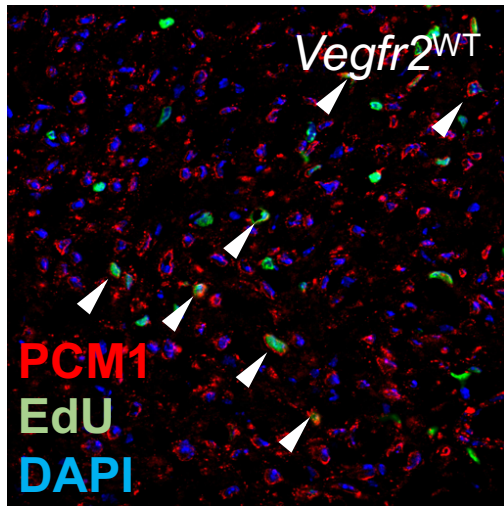

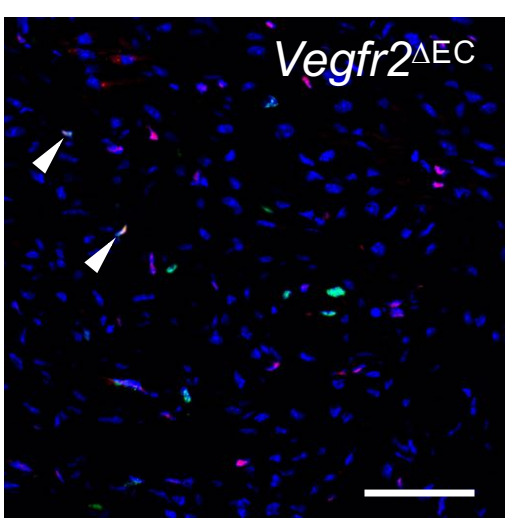

f
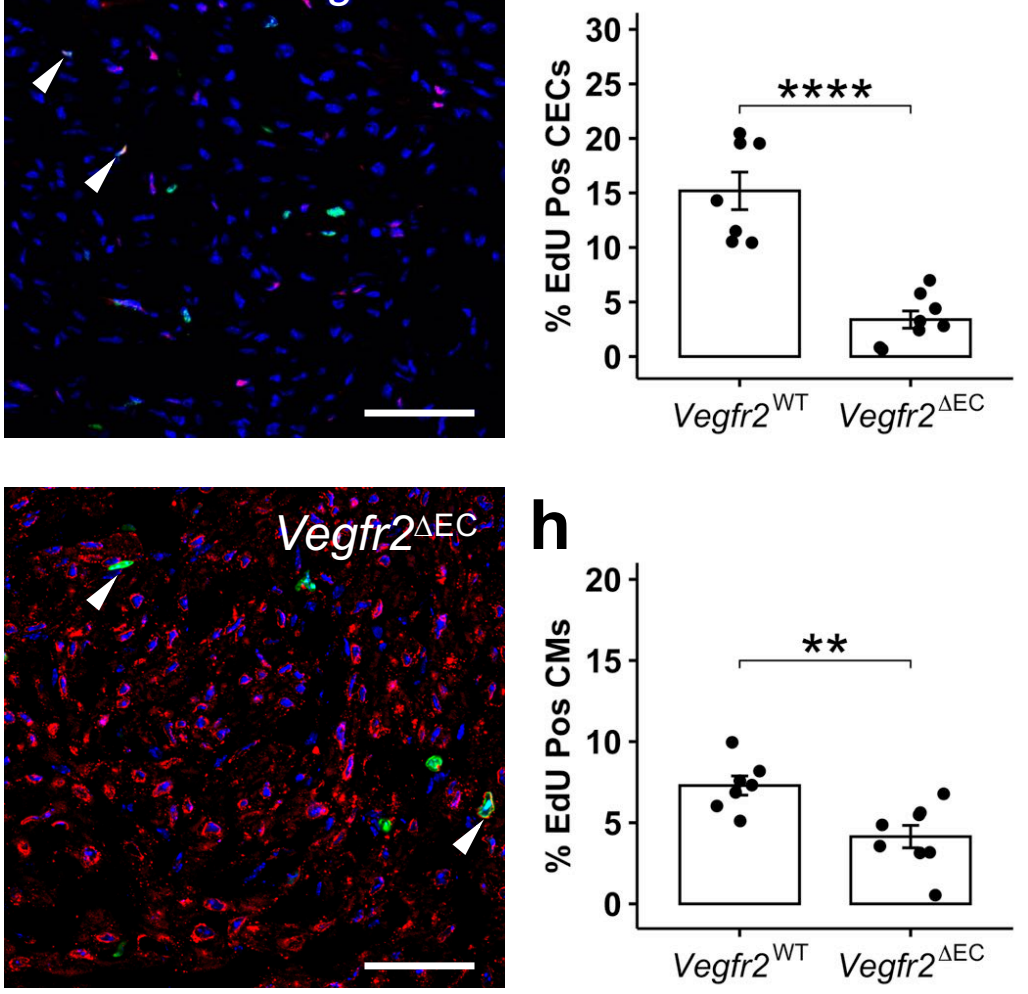
Physiologic Growth
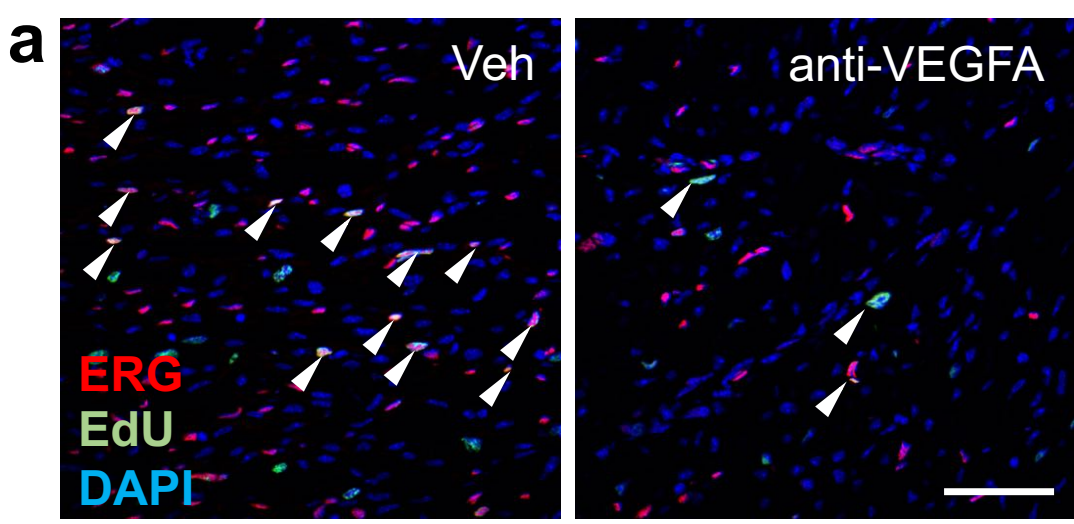

\section{b}

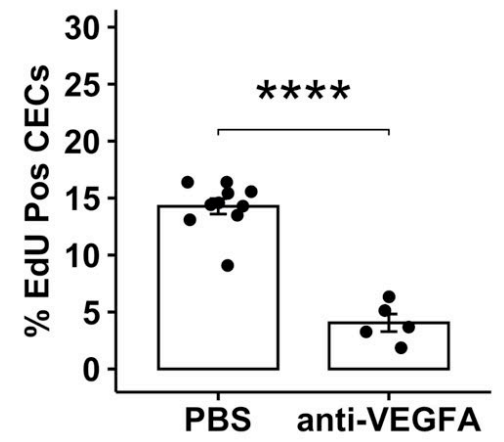

C
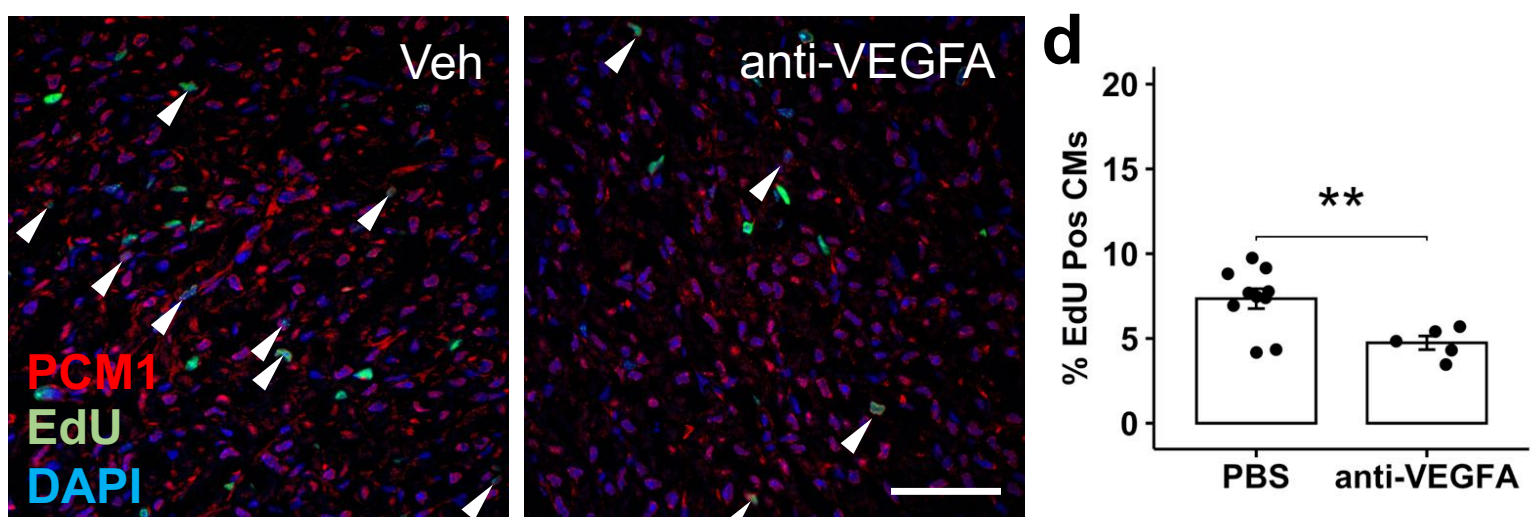

After Injury

e

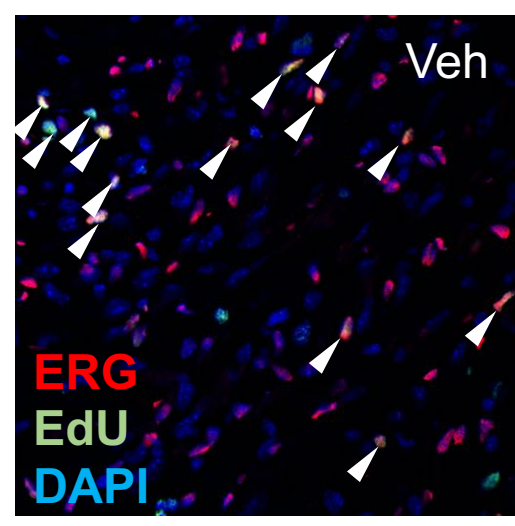

g

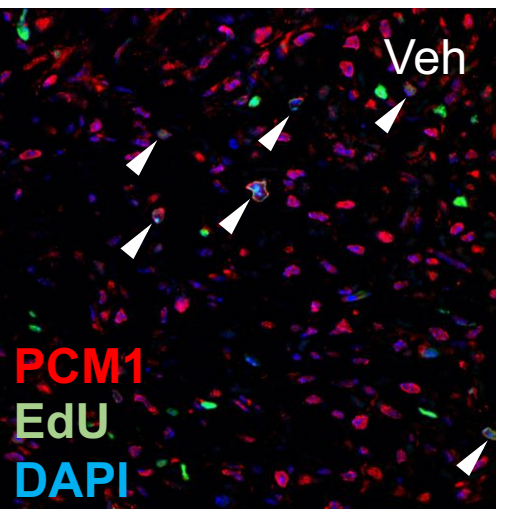

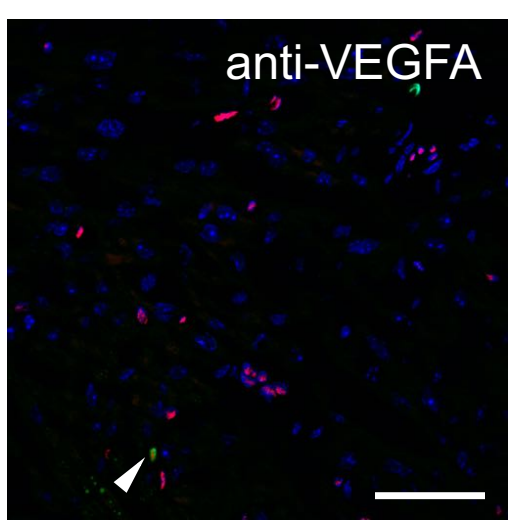
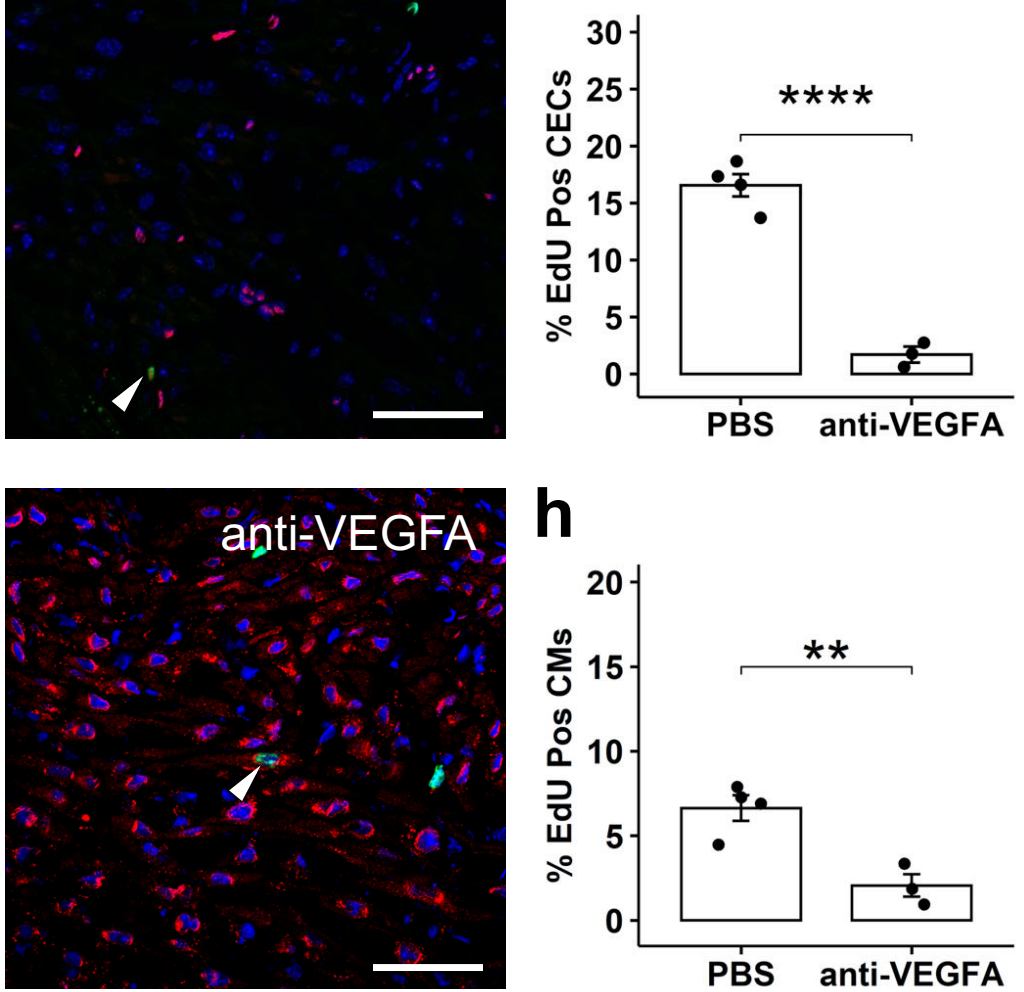

h

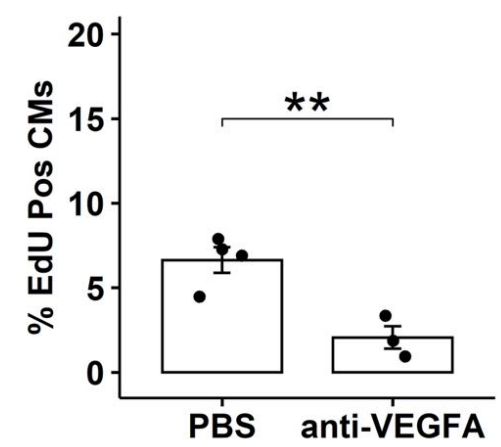




\section{Figure 6}

a
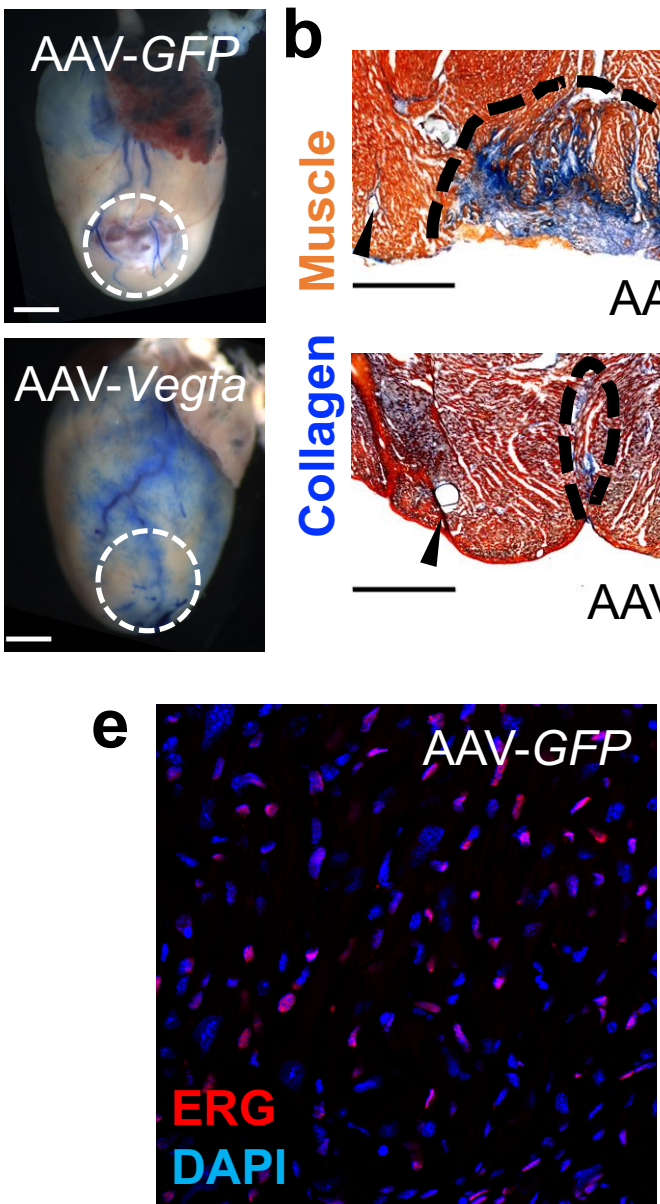

e

g

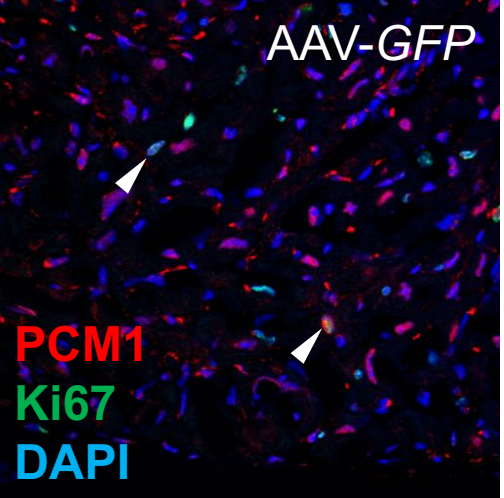

C

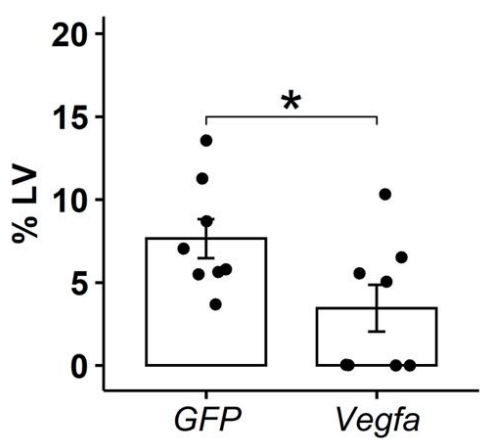

d

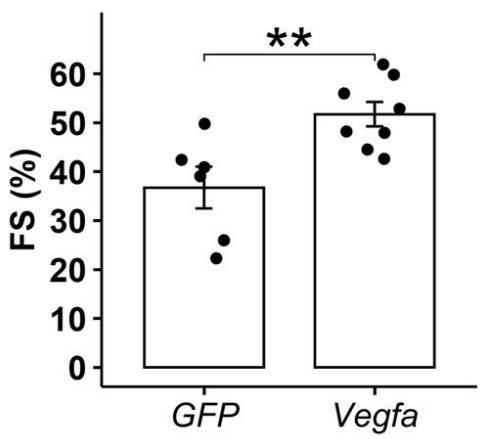

\section{AAV-Vegfa}
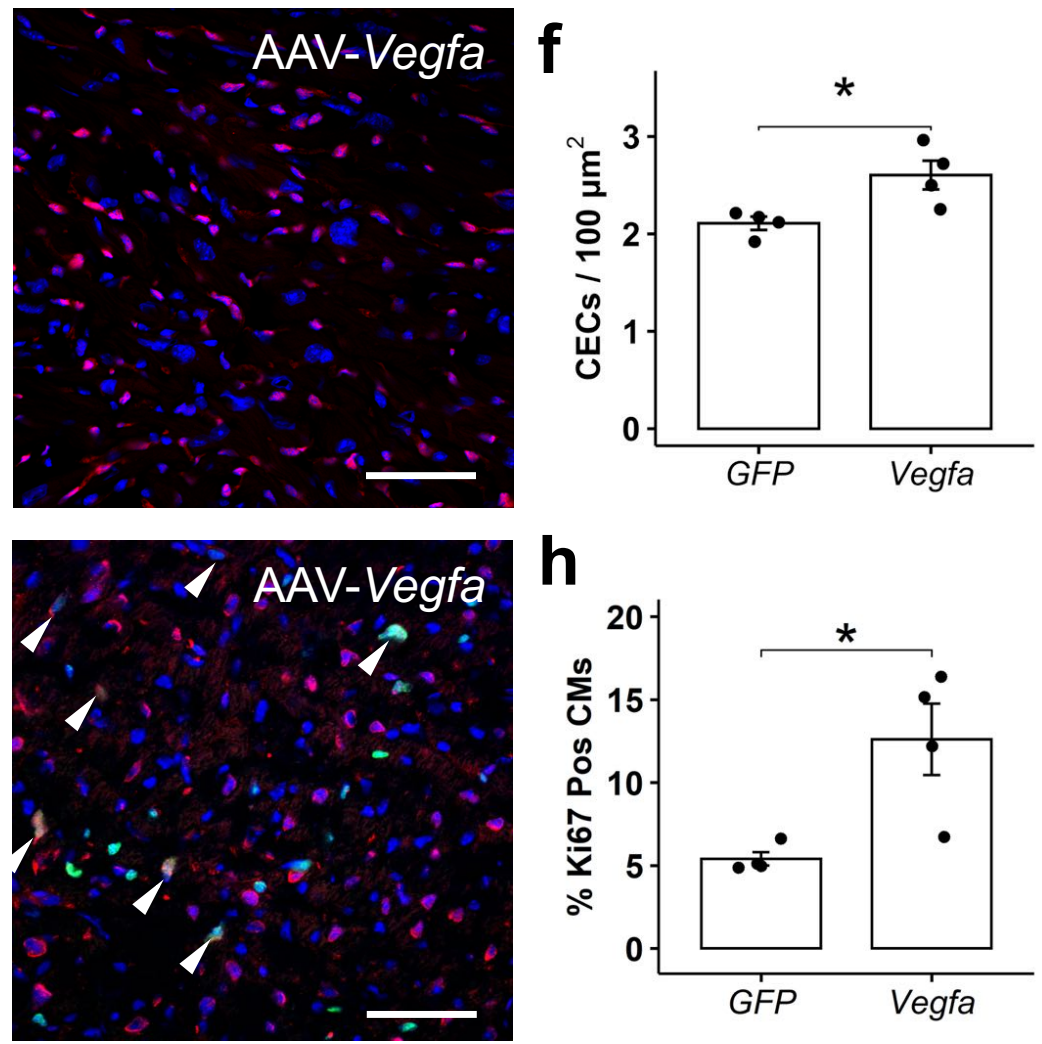
Figure 7

a

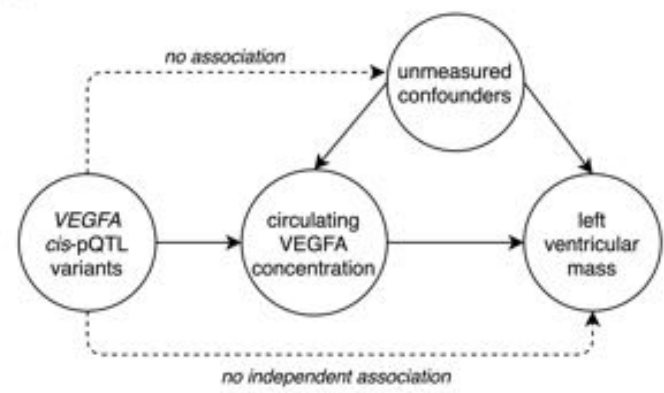

b

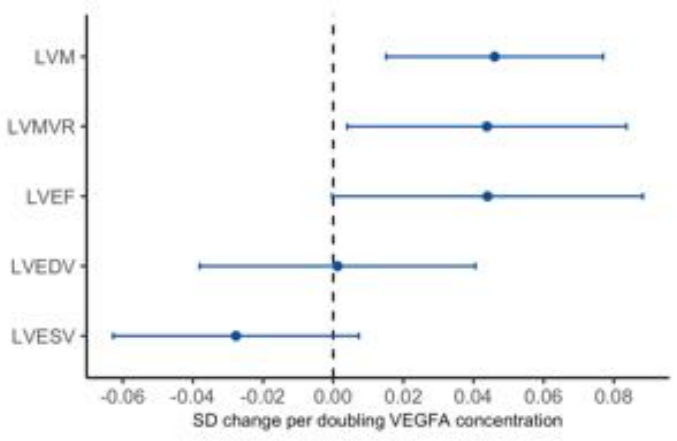

\begin{tabular}{|l|c|}
\hline SD change $[95 \% \mathrm{Cl}]$ & P-value \\
\hline $0.046[0.015,0.077]$ & 0.004 \\
\hline $0.044[0.004,0.084]$ & 0.031 \\
\hline $0.044[-0.000,0.088]$ & 0.052 \\
\hline $0.001[-0.038,0.041]$ & 0.950 \\
\hline$-0.028[-0.063,0.007]$ & 0.121 \\
\hline
\end{tabular}

